\title{
The evidence of genetic polymorphisms of genes involved in the $P 2 R X 7$ signaling pathway as predictive biomarkers for response and loss of response to infliximab against Crohn's disease
}

\author{
Araki $\mathrm{C}^{1,2}$, Yoshimura $\mathrm{M}^{1}$, Fukumitsu $\mathrm{Y}^{1}$, Ma $\mathrm{S}^{1}$, Ishida $\mathrm{T}^{3}$, Urabe $\mathrm{S}^{4}$, Matsushima $\mathrm{K}^{4}$, Honda $\mathrm{T}^{5}$, Uehara $\mathrm{R}^{5}$, Fukuda $\mathrm{Y}^{4}$, Takeshima $\mathrm{F}^{4}$, \\ Higuchi $\mathrm{N}^{2}$, Isomoto $\mathrm{H}^{6}$, Nakao $\mathrm{K}^{4}$ and Tsukamoto $\mathrm{K}^{1 *}$ \\ ${ }^{1}$ Department of Pharmacotherapeutics, Nagasaki University Graduate School of Biomedical Sciences, Nagasaki, Japan \\ ${ }^{2}$ Department of Pharmacy, Nagasaki Harbor Medical Center City Hospital, Shinchi-machi, Nagasaki 850-8555, Japan \\ ${ }^{3}$ Department of Gastroenterology, Oita Red Cross Hospital, Chiyo-machi, Oita, Japan \\ ${ }^{4}$ Department of Gastroenterology and Hepatology, Nagasaki University Graduate School of Biomedical Sciences, Sakamoto, Nagasaki, Japan \\ ${ }^{5}$ Department of Gastroenterology and Hepatology, Nagasaki Harbor Medical Center City Hospital, Shinchi-machi, Nagasaki, Japan \\ ${ }^{6}$ Division of Medicine and Clinical Science, Department of Multidisciplinary Internal Medicine, Faculty of Medicine, Tottori University, Nishi-cho, Yonago, Japan
}

\begin{abstract}
Infliximab (IFX) is a chimeric anti-tumor necrosis factor- $\alpha$ monoclonal antibody exerting the therapeutic effect for Crohn's disease (CD). To identify certain genes related to the effect of IFX and biomarkers to predict the effect of IFX, we examined an association study between 35 tag single nucleotide polymorphisms (SNPs) in six candidate genes involved in the P2RX7 signaling pathway and response to IFX after 10 weeks, 1 year, and 2 years of treatment for Japanese CD patients. A total of $127 \mathrm{CD}$ patients were divided into two groups, including responders and non-responders, at each period of IFX treatment. The frequencies of alleles and genotyped at each tag SNP between responders and non-responders were compared in three different inheritance models at each period of treatment. Statistical analyses indicated that polymorphism of rs11670259 in CARD8 contributed to response and primary non-response to IFX after 10 weeks of treatment, and that polymorphisms of $P 2 R X 7, C A R D 8$, and CASP1 independently contributed to response and secondary loss of response to IFX after 1 year of treatment. Subsequently, using the associated tag SNPs as a biomarker, genetic test revealed that the polymorphism of CARD8 and the combination polymorphisms of P2RX7 and CASP1 were useful as a biomarker to predict response to IFX after 10 weeks and 1 year of treatment, respectively. Therefore, $C A R D 8$ is an IFX-related gene after 10 weeks of treatment, and $P 2 R X 7, C A R D 8$, and CASP1 are IFX-related genes after 1 year of treatment for Japanese CD patients. These genes in the P2RX7 signaling pathway could therefore be potential targets for new therapeutic drugs to combat primary non-response and secondary loss of response to IFX for CD patients.
\end{abstract}

\begin{abstract}
Abbreviations: ASC: apoptosis-associated speck-like protein containing a card; ATI: antibodies to IFX; CARD8: caspase recruitment domain-containing protein 8; CASP1: caspase 1; CD: Crohn's disease; CDAI: Crohn's disease activity index; HWE: Hardy-Weinberg equilibrium; IFX: infliximab; IL: interleukin; NF- $\mathrm{B}$ : nuclear factor kappa-B; NLRP3: NLR family pyrin domain-containing 3; P2RX7: purinergic receptor P2X, ligand-gated ion channel, 7; SNPs: single nucleotide polymorphisms; TNF- $\alpha$ anti-tumor necrosis factor-alpha; TNFR: TNF receptor
\end{abstract}

\section{Introduction}

Crohn's disease $(\mathrm{CD})$ is pathophysiologically characterized by granulomatous inflammation in the gastrointestinal tract with the dysfunction of both the mucosal immune system and inflammatory response. Although the etiology of $\mathrm{CD}$ is still unknown, many genetic and environmental factors contribute to the onset of $\mathrm{CD}$, because this disorder is one of the multifactorial disorders [1-3]. Since no fundamental therapies for $\mathrm{CD}$ have yet been established, $\mathrm{CD}$ treatment is determined according to the present site of the lesions, the degree of inflammation, response to the past treatment, and the presence or absence of complications in order to induce remission as early as possible [4].
Infliximab (IFX) is a chimeric anti-tumor necrosis factor- $\alpha$ (TNF- $\alpha$ ) monoclonal antibody, which is used for the CD patients with moderate to severe disease activity [4-6]. A randomized clinical trial using a $5 \mathrm{mg} / \mathrm{kg}$ intravenous infusion of IFX (ACCENT I) has revealed that $58 \%$ of all patients showed good response to IFX after 2 weeks of treatment. However, among the responders after 2 weeks, $22 \%$ of the patients discontinued maintenance treatment by 54 weeks of the treatment period [7]. Therefore, response to the therapeutic drugs for $\mathrm{CD}$ as well as susceptibility to the onset of $\mathrm{CD}$ are involved as a multifactorial complex.

Some association studies using single nucleotide polymorphisms

Correspondence to: Kazuhiro Tsukamoto, Department of Pharmacotherapeutics, Nagasaki University Graduate School of Biomedical Sciences, Sakamoto, Nagasaki, Japan, E-mail: ktsuka@nagasaki-u.ac.jp

Key words: $P 2 R X 7$ signaling pathway, single nucleotide polymorphism, infliximab, drug-responsibility gene, Crohn's disease, candidate gene-based association study, DNA-based biomarker

Received: December 04, 2016; Accepted: December 26, 2016; Published: December 29, 2016 
Araki C (2016) The evidence of genetic polymorphisms of genes involved in the $P 2 R X 7$ signaling pathway as predictive biomarkers for response and loss of response to infliximab against Crohn's disease

(SNPs) have shown the possible IFX-related genes for rheumatoid arthritis, such as TNF receptor (TNFR) superfamily member $1 \mathrm{~B}$ [8], Fc gamma receptors IIA and IIIA [9], AFF3 [10], CD226 [10], protein tyrosine phosphatase receptor type C [11], and p38 mitogen-activated protein kinase [12]. While, our previous study has reported that the polymorphisms of IL17F and TRAF3IP2 are associated with response to IFX after 1 year of treatment for Japanese CD patients [13]. As the results, we next focused on the purinergic receptor $\mathrm{P} 2 \mathrm{X}$, ligand-gated ion channel, 7 (P2RX7/P2RX7) signaling pathway, which is related to intestinal inflammation through another non-TNF-dependent inflammation signaling pathway.

P2RX7 is a ligand-gated membrane ion channel which plays a crucial role in many cellular functions, such as vascular reactivity, apoptosis, cytokine secretion, and tissue inflammation [14]. This purinergic receptor is expressed in peripheral macrophages, mast cells, lymphocytes, erythrocytes, fibroblasts, microglia, astrocytes, Schwann cells, and dendritic cells, and is activated by high concentrations of extracellular ATP [15], thus resulting in the opening of ion channels and subsequently leading to the influx of $\mathrm{Ca}^{2+}$ and the efflux of $\mathrm{K}^{+}$. The increase in cytosolic $\mathrm{Ca}^{2+}$ and subsequent decrease in intracellular $\mathrm{K}^{+}$activate the assembly of caspase recruitment domain-containing protein 8 (CARD8/CARD8), NLR family pyrin domain-containing 3 (NLRP3/NLRP3), apoptosis-associated speck-like protein containing a card (ASC/PYCARD), and caspase 1 (CASP1/CASP1)[16], thus resulting in the release of inflammatory cytokines including interleukin (IL)-1 $\beta$ (IL1B/IL1B) $[16,17]$, IL-2, IL-4, IL-6, IL-13, and IL-18 (IL18) [17], and other inflammatory mediators, such as nitric oxide synthase, cycloxygenase-2, TNF- $\alpha$ [18], phospholipase- $D$, phospholipase $A_{2}$, nuclear factor kappa-B (NF-kB)[19], and mitogen activated protein kinases. Since the P2RX7 signaling pathway regulates inflammation through the secretion of these cytokines and mediators, P2RX7 antagonists can be used in the treatment of inflammatory bowel disease [20]. In addition, the production of IL- $1 \beta$ was found to decrease in caspase1-deficient mice as well as in P2RX7-deficient mice [21-23]. Moreover, NLRP3 and CARD 8 are susceptibility genes for the onset of $\mathrm{CD}$ [24]. The number of mast cells expressing $P 2 R X 7$ have been reported to increase in the colon of CD patients and intestinal inflammation is inhibited by the treatment of anti-P2RX7 antibody [25].

We therefore hypothesized that not only the activation of the TNFR signaling pathway, but also the activation of the P2RX7 signaling pathway may contribute to inflammation of the intestines in CD patients. The elevated production of inflammatory cytokines and mediators through the activation of the P2RX7 signaling pathway in the genetic background may lead to the perpetuation of the chronic intestinal inflammatory process and might thereby result in loss of response to IFX. Therefore, we examined an association study between polymorphisms of six target genes (P2RX7, CARD8, PYCARD, $C A S P 1, I L 1 B$, and $I L 18$ ) involved in the P2RX7 signaling pathway and the therapeutic effect of IFX at the short, middle, or long period of treatment for Japanese CD patients. Another purpose of this study was to investigate whether the associated polymorphisms can be used as new genetic biomarkers for predicting either response or loss of response to IFX at each period by genetic testing.

\section{Patients and methods}

\section{Patients}

In this study, 127 unrelated Japanese CD patients were enrolled and treated with IFX at three general hospitals, namely Oita Red Cross Hospital, Nagasaki Harbor Medical Center City Hospital, or Nagasaki
University Hospital from 2004 to 2012.

The study protocol was approved by the Ethics Committee dealing with Human Genome and Gene Analysis at Oita Red Cross Hospital, Nagasaki Harbor Medical Center City Hospital, and Nagasaki University. Written informed consent was obtained from all patients.

Definition of the therapeutic effect of IFX: Since a higher Crohn's disease activity index (CDAI) of more than 150 is regarded as activephase CD patients [26], responders to IFX were defined as those showing a decrease in CDAI of less than 150 and an improvement in clinical manifestations, laboratory data, and/or endoscopic findings at each period of treatment. Non-responders to IFX were defined as those showing no change in the CDAI value or any exacerbation of the disease activity.

\section{Study design}

All enrolled patients were analyzed after 10 weeks of treatment. Out of the $127 \mathrm{CD}$ patients, 116 patients were subsequently analyzed after 1 year of treatment because they showed response to IFX after 10 weeks of treatment. Thus, 11 patients indicated primary non-response to IFX. Likewise, out of 116 patients, 97 patients, who had shown response to IFX after 1 year of treatment, were subjected to an analysis after 2 years of treatment. Therefore, 19 patients indicated secondary loss of response to IFX after 1 year of treatment. The clinical characteristics of the patients in each group at the end of this study are shown in Table 1.

Selection of tag SNPs in the candidate genes: The selected six candidate genes involved in the P2RX7 signaling pathway included P2RX7 (OMIM \#602566) located at 12q24.31; CARD8 (OMIM \#609051) located at 19q13.33; PYCARD (OMIM \#606838) located at 16p11.2; CASP1 (OMIM \#147678) located at 11q22.3; IL18 (OMIM \#600953) located at 11q23.1, and IL1B (OMIM \#147720) located at $2 \mathrm{q} 14.1$.

Obtaining information on SNPs in the target genes, selecting the candidate tag SNPs, and determining the genotyped tag SNPs were carried out according to the same methods as reported previously $[13,27,28]$. The gene structures and positions of the genotyped tag SNPs in the candidate genes are shown in Figure 1.

Genotyping of tag SNPs in candidate genes: Genetic analyses for the genomic DNA extracted from each patient and genotyping of 35 tag SNPs in 6 genes by PCR-restriction fragment length polymorphism, PCR-direct DNA sequencing, or probe-based high resolution melting method were done according to the same method as reported

Table 1. Comparison of the characteristics between responders and non-responders to IFX in CD patients. (IFX, infliximab; CD, Crohn's disease; SD, standard deviation).

\begin{tabular}{|c|c|c|}
\hline \multirow{2}{*}{ Characteristics } & \multicolumn{2}{|c|}{ Number (\%) of CD patients } \\
\cline { 2 - 3 } & Responders & Non-responders \\
\hline 10 weeks $(n=127)$ & & \\
\hline Number (\%) & $116(91.3)$ & $11(8.7)$ \\
\hline Age, mean \pm SD (years) & $35.2 \pm 11.8$ & $35.3 \pm 11.8$ \\
\hline Male/female (\%) & $67 / 49(57.8 / 42.2)$ & $10 / 1(90.9 / 9.1)$ \\
\hline 1 year $(n=116)$ & & \\
\hline Number (\%) & $97(83.6)$ & $19(16.4)$ \\
\hline Age, mean \pm SD (years) & $35.2 \pm 11.9$ & $35.6 \pm 11.7$ \\
\hline Male/female (\%) & $56 / 41(57.7 / 42.3)$ & $11 / 8(57.9 / 42.1)$ \\
\hline 2 years $(n=97)$ & & $15(15.5)$ \\
\hline Number (\%) & $82(84.5)$ & $35.3 \pm 11.8$ \\
\hline Age, mean \pm SD (years) & $35.1 \pm 11.9$ & $8 / 7(53.3 / 46.7)$ \\
\hline Male/female (\%) & $48 / 34(58.5 / 41.5)$ & \\
\hline
\end{tabular}



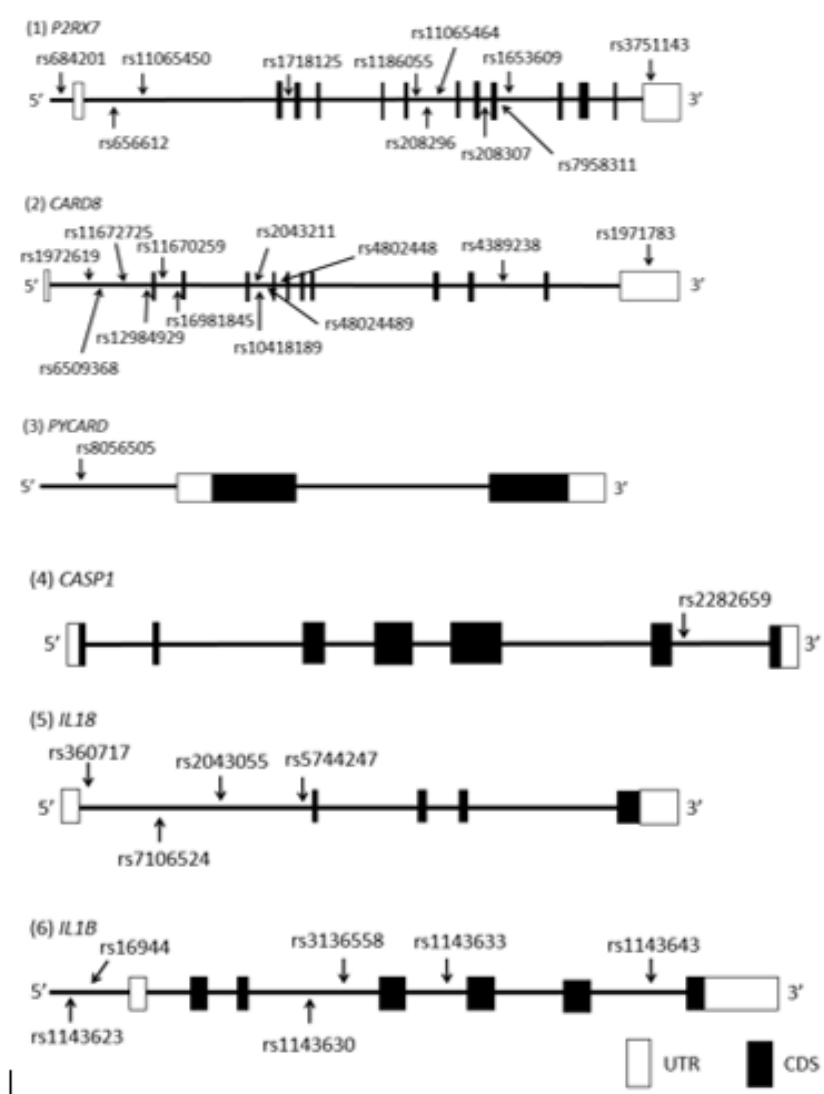

Figure 1. Gene structures and locations of the genotyped tag SNPs in each gene. The horizontal bars indicate the genomic sequences of the candidate genes. Full boxes represent exons in each gene, and open boxes show the untranslated regions. The arrows indicate the positions of the genotyped tag SNP sites and their names are presented above each site.

previously $[13,27,28]$. Information on the sequences of primer sets, annealing temperature, cycle number, restriction enzymes, and genetic analytic methods for each SNP is shown in Table 2.

\section{Statistical analysis}

A comparison of the clinical characteristics, the significance of deviation from the Hardy-Weinberg equilibrium (HWE), and the frequencies of alleles and genotypes, subsequent multivariate regression analysis, and the statistical value of genetic tests were analyzed by the same methods as reported previously $[13,27,28]$.

\section{Results}

\section{Comparison of the clinical characteristics between responders and non-responders to IFX in each group}

Comparison analyses of the clinical characteristics between responders and non-responders to IFX in each group revealed a significant difference in the gender after 10 weeks of treatment. The percentage of males in non-responders to IFX was higher than that in responders ( $90.9 \%$ vs. $57.8 \%, P=0.049$; Table 1$)$, thereby indicating that male gender showed loss of response to IFX after 10 weeks of treatment. Conversely, females indicated response to IFX after 10 weeks of treatment. However, no significant differences in the mean age and gender were observed between responders and non-responders to IFX in other groups.

\section{Association of tag SNPs with response to IFX after 10 weeks of} treatment

The frequencies and distributions of minor alleles and genotypes at tag SNPs in each gene were identified and compared between responders and non-responders to IFX after 10 weeks of treatment (Table 3). The two tag SNPs, rs2043211 and rs1972619 in CARD8, were excluded from the subsequent analyses because they were not in HWE.

The frequencies of a heterozygous $\mathrm{C} / \mathrm{T}$ genotype and a minor homozygous $\mathrm{T} / \mathrm{T}$ genotype of rs11670259 in CARD8 in the minor allele dominant model were significantly decreased in responders in comparison to those in non-responders $(31.9 \%$ vs. $63.6 \%, P=0.047$, $\mathrm{OR}=0.268$; Table 3 ), thereby indicating $\sim 3.7$-fold loss of response to IFX after 10 weeks of treatment. Conversely, the possession of a major homozygous C/C genotype of rs11670259 in CARD8 indicated 3.7fold response to IFX.

Moreover, the frequencies of a heterozygous G/C genotype and a minor homozygous C/C genotype of rs1143623 in IL1B in the minor allele dominant model were significantly decreased in responders in comparison to those in non-responders $(55.2 \%$ vs. $90.9 \%, P=0.025$, $\mathrm{OR}=0.123$; Tables 3 ), indicating that this genotype is associated with $\sim 8.1$-fold loss of response to IFX. Conversely, the possession of a major homozygous G/G genotype of rs1143623 in IL1B indicated $~ 8.1$-fold response to IFX after 10 weeks of treatment.

There were no significant differences in the frequencies of any other alleles and genotypes at tag SNPs between responders and nonresponders after 10 weeks of treatment.

\section{Association of tag SNPs with response to IFX after 1 year of treatment}

The frequencies and distributions of minor alleles and genotypes at tag SNPs in each gene were identified and compared between responders and non-responders to IFX after 1 year of treatment (Table 4).

With regard to rs3751143 in $P 2 R X 7$, the frequency of a minor homozygous $G / G$ genotype in the minor allele recessive model were significantly lower in responders in comparison to those in nonresponders $(6.2 \%$ vs. $31.6 \%, P=0.001$, OR $=0.143$; Table 4$)$. This result implied that $\sim 7.0$-fold loss of response to IFX after 1 year of treatment. Conversely, the possession of a major homozygous $\mathrm{T} / \mathrm{T}$ genotype or a heterozygous $\mathrm{T} / \mathrm{G}$ genotype of $\mathrm{rs} 3751143$ indicated $\sim 7.0$-fold response to IFX.

Moreover, the frequencies of a heterozygous $\mathrm{C} / \mathrm{T}$ genotype and a minor homozygous $\mathrm{T} / \mathrm{T}$ genotype of rs 4389238 in CARD 8 in the minor allele dominant model were significantly decreased in responders in comparison to those in non-responders $(46.4 \%$ vs. $78.9 \%, P=0.012$, $\mathrm{OR}=0.231$; Tables 4 ), indicating that these genotypes are associated with $\sim 4.3$-fold loss of response to IFX. Conversely, the possession of a major homozygous C/C genotype of rs4389238 in CARD8 indicated $\sim 4.3$-fold response to IFX after 1 year of treatment.

In addition, the frequencies of a heterozygous $A / G$ genotype and a minor homozygous $\mathrm{G} / \mathrm{G}$ genotype of rs2282659 in CASP1 in the minor allele dominant model were significantly increased in responders in comparison to those in non-responders $(55.7 \%$ vs. $15.8 \%, P=0.002$, $\mathrm{OR}=6.698$; Tables 4 ), indicating that these genotypes are associated with $\sim 6.7$-fold response to IFX. Conversely, possessing a major homozygous A/A genotype of rs2282659 in CASP1 indicated $~ 6.7$-fold loss of response to IFX after 1 year of treatment. 
Araki C (2016) The evidence of genetic polymorphisms of genes involved in the $P 2 R X 7$ signaling pathway as predictive biomarkers for response and loss of response to infliximab against Crohn's disease

Table 2. Information on genotyping of tag SNPs in the candidate genes (SNP, single nucleotide polymorphism; 3'-UTR, 3'-untranslated region; PCR, polymerase chain reaction; RFLP, restriction fragment length polymorphism; HRM, high resolution melting).

\begin{tabular}{|c|c|c|c|c|c|c|c|}
\hline \multirow[t]{2}{*}{ Gene } & \multirow[t]{2}{*}{ tag SNP } & \multirow{2}{*}{$\begin{array}{l}\text { Major }> \\
\text { Minor }\end{array}$} & \multicolumn{2}{|c|}{ Sequence of primer (5' to 3') } & \multirow{2}{*}{$\begin{array}{c}\text { Annealing } \\
\text { temperature } \\
\left({ }^{\circ} \mathrm{C}\right)\end{array}$} & \multirow{2}{*}{$\begin{array}{c}\text { Cycle } \\
\text { number }\end{array}$} & \multirow{2}{*}{$\begin{array}{c}\text { Analytic method (Restriction } \\
\text { enzyme) }\end{array}$} \\
\hline & & & Forward & Reverse & & & \\
\hline \multirow[t]{11}{*}{$P 2 R X 7$} & rs684201 & $\mathrm{G}>\mathrm{A}$ & ATCTGATTTCCCCCACCAAC & GACTGGGAGCTTCCATTATGC & 55 & 30 & PCR-RFLP (BsrD I) \\
\hline & rs656612 & $\mathrm{A}>\mathrm{C}$ & GCAATTGCTGACCCCCTATT & CAACAGCTTGGTGGTCACAG & 56 & 30 & PCR-RFLP (Cac8 I) \\
\hline & rs 11065450 & $\mathrm{C}>\mathrm{A}$ & GTGCCGGGTTCTGTTCTTAG & GCTTGGGTCTCCTGTTGTGT & 57 & 50 & PCR-HRM \\
\hline & rs 1718125 & $\mathrm{G}>\mathrm{A}$ & GGCTGGTGCTCTTTGGTAGA & GGGTGAGATCCAGGAGATGA & 57 & 30 & PCR-RFLP (Bsh1236 I) \\
\hline & rs1186055 & $\mathrm{G}>\mathrm{T}$ & GAAGATCTGGGGGAAGGAAG & GCTTGGCACAAACTAGTATCTCTGG & 56 & 50 & PCR-HRM \\
\hline & rs208296 & $\mathrm{C}>\mathrm{T}$ & AGCCATTTTCCCAAGGACAC & GGGGGAAGGAAGTTTTCTCA & 55 & 30 & PCR-RFLP (Xsp I) \\
\hline & rs11065464 & $\mathrm{C}>\mathrm{A}$ & GGCAAGCAACTCCCTGAACT & GGCATTTACTGTGGCACCTC & 58 & 30 & PCR-RFLP (Mae III) \\
\hline & rs208307 & $\mathrm{G}>\mathrm{C}$ & TGGGACACTGTGGATTCTGA & AGGAGGCAGTGATCATTTGG & 55 & 30 & PCR-RFLP (HpyCH4 V) \\
\hline & rs7958311 & $\mathrm{G}>\mathrm{A}$ & AACGGTGATGTGTCCCAGAC & CCACTGGGTGAGTTTGACCT & 57 & 30 & PCR-RFLP $($ Fok I) \\
\hline & rs1653609 & $\mathrm{A}>\mathrm{C}$ & CACCCATAATTCCCCTACCC & AGAATTTTGAGTGGCTGTGG & 54 & 30 & PCR-RFLP (Mbo I) \\
\hline & rs 3751143 & $\mathrm{~T}>\mathrm{G}$ & TGTCTGGACAGGACCAGCTT & TTCCTGGACAACCAGAGGAG & 59 & 30 & PCR-RFLP (Hha I) \\
\hline \multirow[t]{12}{*}{ CARD8 } & rs1971783 & $\mathrm{T}>\mathrm{C}$ & CCAATAGCTTATATGCCCAGAAGG & CAGGTGAACACTCCAGCAAAT & 55 & 30 & PCR-RFLP (BsaA I) \\
\hline & rs4389238 & $\mathrm{C}>\mathrm{T}$ & AATAGGGTTCGCGCTCCTAC & TGCCCAGGAAGAAGGACATA & 57 & 30 & PCR-RFLP (Fau I) \\
\hline & rs 4802448 & $\mathrm{G}>\mathrm{A}$ & ACAGGTGCTGTTGGGATACAG & TCCAGTCCTCAGCAAATGGT & 57 & 50 & PCR-HRM \\
\hline & rs4802449 & $\mathrm{G}>\mathrm{A}$ & ACTTGACCACACCTGGGAAG & GCTACCCGAATCCATAGCAA & 57 & 30 & PCR-RFLP $(D d e$ I) \\
\hline & rs10418189 & $\mathrm{G}>\mathrm{A}$ & ACAGCGCATCCCAGATCAT & ACCTCAAGGGCATAGACTGCT & 57 & 30 & PCR-direct DNA sequencing \\
\hline & rs2043211 & $\mathrm{A}>\mathrm{T}$ & CCCCTGAGTTCGATGAAAAA & TAGGGGCCTGAGGAATGACT & 53 & 30 & PCR-RFLP $(M b o$ I) \\
\hline & rs16981845 & $\mathrm{T}>\mathrm{C}$ & CTCAGACTCCCATGACTCTTTCTT & TCACTAATGCCCTGCAATCC & 55 & 30 & PCR RFLP (Hsp92 II) \\
\hline & rs11670259 & $\mathrm{C}>\mathrm{T}$ & CCATGGGAGTTTTCCCTTCA & CCGAGACGGTGAGTGACTTT & 56 & 50 & PCR-HRM \\
\hline & rs12984929 & $\mathrm{G}>\mathrm{T}$ & CCAACCTGACAGAGGCAAAA & GAGGGTGGACACATACATTCG & 56 & 30 & PCR-RFLP (Hinc II) \\
\hline & rs11672725 & $\mathrm{C}>\mathrm{T}$ & CCCTGGCCCAGAGAAATAAT & CGCAGGCTGTGAGAAGCATA & 56 & 50 & PCR-HRM \\
\hline & rs6509368 & $\mathrm{G}>\mathrm{A}$ & TCAACCAGCTTATCTGAATGTTAGC & GTTGACCATGTTCCGCAAA & 55 & 30 & PCR-RFLP $(A f a$ I $)$ \\
\hline & rs1972619 & $\mathrm{G}>\mathrm{A}$ & TGGAAGCATTGTGTGTGTGTG & ACTGTGTTTGGGGAGCAGAC & 56 & 30 & PCR-RFLP (Hpy8 I) \\
\hline PYCARD & rs 8056505 & $\mathrm{~T}>\mathrm{C}$ & AGGGGAGCCAGAATTTGATC & CCTCCACCACACACAGCTAT & 56 & 30 & PCR-RFLP (НpyCH4 IV) \\
\hline CASPl & rs 2282659 & $\mathrm{~A}>\mathrm{G}$ & GGAGCGGGGTGAAACTAAAT & CCACAATGGGCTCTGTTTTT & 54 & 30 & PCR-RFLP (Ban II) \\
\hline \multirow[t]{4}{*}{ IL18 } & rs5744247 & $\mathrm{C}>\mathrm{G}$ & GAGAAAGAGGGCCACCAAAT & TGGCCTAGTACTGAACTGAATC & 54 & 30 & PCR-RFLP (Ple I) \\
\hline & rs2043055 & $A>G$ & CCTCCACCTGAAAGCCAAT & TCCTGGGAGGATCTTTCTGA & 55 & 30 & PCR-RFLP (Rsa I) \\
\hline & rs 7106524 & $\mathrm{G}>\mathrm{A}$ & GTGGCTGAACTCACCACAGA & CTTTCAGGCCAGGTGCACTA & 57 & 50 & PCR-HRM \\
\hline & rs 360717 & $\mathrm{C}>\mathrm{G}$ & CCATGGCTGACTTTCCAAAT & GCACAGAGCCCCAACTTTTA & 54 & 30 & PCR-RFLP $(A c i$ I) \\
\hline \multirow[t]{6}{*}{$I L I B$} & rs 1143643 & $\mathrm{G}>\mathrm{A}$ & AAAAGCCCCTGGAAACTAGG & CACATGATCAGGAGCCAGAC & 54 & 50 & PCR-HRM \\
\hline & rs 1143633 & $\mathrm{~A}>\mathrm{G}$ & TGTTCTTAGCCACCCCACTC & TCCATATCCTGTCCCTGGAG & 56 & 30 & PCR-RFLP (Fun4H I) \\
\hline & rs 3136558 & $\mathrm{~T}>\mathrm{C}$ & GGTGTCAGAAAGCCCACATT & GAGGAGGAAAGGGCTTGAAA & 55 & 30 & PCR-RFLP (Mbo I) \\
\hline & rs 1143630 & $\mathrm{C}>\mathrm{A}$ & AGGTGGGGCATGTACAAAAA & AGATTATCCCTCTCTGAAGCTC & 54 & 50 & PCR-HRM \\
\hline & rs16944 & $\mathrm{G}>\mathrm{A}$ & GCCCTCCCTGTCTGTATTGA & AGGCACTTTGCTGGTGTCTC & 56 & 30 & PCR-RFLP (Ava I) \\
\hline & rs 1143623 & $\mathrm{G}>\mathrm{C}$ & ATCAGAAGGCTGCTTGGAGA & AAACCTTGCTCCTCCTGGTT & 56 & 30 & PCR-RFLP (BseD I) \\
\hline
\end{tabular}

Table 3. Allele and genotype comparisons in three inheritance models between responders and non-responders to IFX after 10 weeks of treatment for CD patients.

\begin{tabular}{|c|c|c|c|c|c|c|c|c|}
\hline \multirow[t]{2}{*}{ Gene } & \multirow{2}{*}{$\begin{array}{c}\text { Tag SNP } \\
\text { (Major }>\text { Minor) }\end{array}$} & \multirow[t]{2}{*}{ Genotype } & \multicolumn{2}{|c|}{ Number (\%) of CD patients } & \multirow[t]{2}{*}{ Inheritance model* } & \multirow[t]{2}{*}{$P$ value } & \multirow[t]{2}{*}{ OR } & \multirow[t]{2}{*}{$95 \% \mathrm{CI}$} \\
\hline & & & $\begin{array}{c}\text { Responders } \\
(n=116)\end{array}$ & $\begin{array}{c}\text { Non-responders } \\
\quad(n=11)\end{array}$ & & & & \\
\hline \multirow[t]{21}{*}{$P 2 R X 7$} & rs684201 & MAF & 0.211 & 0.182 & Allele & 1.000 & 1.205 & $0.390-3.724$ \\
\hline & $\mathrm{G}>\mathrm{A}$ & $\mathrm{G} / \mathrm{G}$ & $72(62.1)$ & $7(63.6)$ & & & & \\
\hline & & $\mathrm{G} / \mathrm{A}$ & $39(33.6)$ & $4(36.4)$ & Dominant & 1.000 & 1.069 & $0.296-3.864$ \\
\hline & & $\mathrm{A} / \mathrm{A}$ & $5(4.3)$ & $0(0)$ & Recessive & 1.000 & 1.135 & $0.059-21.87$ \\
\hline & rs656612 & MAF & 0.306 & 0.409 & Allele & 0.320 & 0.637 & $0.260-1.558$ \\
\hline & $\mathrm{A}>\mathrm{C}$ & $\mathrm{A} / \mathrm{A}$ & $52(48.8)$ & $3(27.3)$ & & & & \\
\hline & & $\mathrm{A} / \mathrm{C}$ & $57(49.1)$ & $7(63.6)$ & Dominant & 0.347 & 0.462 & $0.117-1.828$ \\
\hline & & $\mathrm{C} / \mathrm{C}$ & $7(6.0)$ & $1(9.1)$ & Recessive & 0.526 & 0.642 & $0.072-5.757$ \\
\hline & rs 11065450 & MAF & 0.332 & 0.409 & Allele & 0.465 & 0.718 & $0.294-1.752$ \\
\hline & $\mathrm{C}>\mathrm{A}$ & $\mathrm{C} / \mathrm{C}$ & $51(44.0)$ & $3(27.3)$ & & & & \\
\hline & & $\mathrm{C} / \mathrm{A}$ & $53(45.7)$ & $7(63.6)$ & Dominant & 0.352 & 0.478 & $0.121-1.894$ \\
\hline & & $\mathrm{A} / \mathrm{A}$ & $12(10.3)$ & $1(9.1)$ & Recessive & 1.000 & 1.154 & $0.136-9.814$ \\
\hline & rs1718125 & MAF & 0.263 & 0.273 & Allele & 0.921 & 0.951 & $0.356-2.541$ \\
\hline & $\mathrm{G}>\mathrm{A}$ & $\mathrm{G} / \mathrm{G}$ & $63(54.3)$ & $5(45.5)$ & & & & \\
\hline & & $\mathrm{G} / \mathrm{A}$ & $45(38.8)$ & $6(54.5)$ & Dominant & 0.754 & 0.701 & $0.203-2.427$ \\
\hline & & $\mathrm{A} / \mathrm{A}$ & $8(6.9)$ & $0(0)$ & Recessive & 1.000 & 1.802 & $0.097-33.31$ \\
\hline & rs 1186055 & MAF & 0.392 & 0.590 & Allele & 0.070 & 0.447 & $0.184-1.088$ \\
\hline & $\mathrm{G}>\mathrm{T}$ & $\mathrm{G} / \mathrm{G}$ & $42(36.2)$ & $1(9.1)$ & & & & \\
\hline & & $\mathrm{G} / \mathrm{T}$ & $57(49.1)$ & $7(63.6)$ & Dominant & 0.097 & 0.176 & $0.022-1.425$ \\
\hline & & $\mathrm{T} / \mathrm{T}$ & $17(14.7)$ & $3(27.3)$ & Recessive & 0.378 & 0.458 & $0.110-1.900$ \\
\hline & rs208296 & MAF & 0.319 & 0.318 & Allele & 0.994 & 1.004 & $0.393-2.566$ \\
\hline
\end{tabular}


Araki C (2016) The evidence of genetic polymorphisms of genes involved in the $P 2 R X 7$ signaling pathway as predictive biomarkers for response and loss of response to infliximab against Crohn's disease

\begin{tabular}{|c|c|c|c|c|c|c|c|c|}
\hline & $\mathrm{C}>\mathrm{T}$ & $\mathrm{C} / \mathrm{C}$ & $51(44.0)$ & $4(36.4)$ & & & & \\
\hline & & $\mathrm{C} / \mathrm{T}$ & $56(48.3)$ & $7(63.6)$ & Dominant & 0.756 & 0.728 & $0.202-2.625$ \\
\hline & & $\mathrm{T} / \mathrm{T}$ & $9(7.8)$ & $0(0.0)$ & Recessive & 1.000 & 2.033 & $0.111-37.27$ \\
\hline & rs11065464 & MAF & 0.237 & 0.045 & Allele & 0.055 & 6.527 & $0.858-49.51$ \\
\hline & $\mathrm{C}>\mathrm{A}$ & $\mathrm{C} / \mathrm{C}$ & $67(57.8)$ & $10(90.9)$ & & & & \\
\hline & & $\mathrm{C} / \mathrm{A}$ & $43(37.1)$ & $1(9.1)$ & Dominant & 0.049 & 7.315 & $0.906-59.17$ \\
\hline & & $\mathrm{A} / \mathrm{A}$ & $6(5.2)$ & $0(0.0)$ & Recessive & 1.000 & 1.353 & $0.071-25.61$ \\
\hline & rs208307 & MAF & 0.147 & 0.318 & Allele & 0.205 & 0.536 & $0.201-1.425$ \\
\hline & $\mathrm{G}>\mathrm{C}$ & $\mathrm{G} / \mathrm{G}$ & $86(74.1)$ & $6(54.5)$ & & & & \\
\hline & & $\mathrm{G} / \mathrm{C}$ & $26(22.4)$ & $3(27.3)$ & Dominant & 0.174 & 0.419 & $0.119-1.472$ \\
\hline & & $\mathrm{C} / \mathrm{C}$ & $4(3.4)$ & $2(18.2)$ & Recessive & 0.085 & 0.161 & $0.026-1.000$ \\
\hline & rs7958311 & MAF & 0.349 & 0.182 & Allele & 0.156 & 2.414 & $0.790-7.375$ \\
\hline & $\mathrm{G}>\mathrm{A}$ & $\mathrm{G} / \mathrm{G}$ & $53(45.7)$ & $8(72.7)$ & & & & \\
\hline & & $\mathrm{G} / \mathrm{A}$ & $45(38.8)$ & $2(18.2)$ & Dominant & 0.117 & 3.170 & $0.800-12.55$ \\
\hline & & $\mathrm{A} / \mathrm{A}$ & $18(15.5)$ & $1(9.1)$ & Recessive & 1.000 & 1.837 & $0.221-15.24$ \\
\hline & rs1653609 & MAF & 0.280 & 0.364 & Allele & 0.408 & 0.681 & $0.273-1.700$ \\
\hline & $\mathrm{A}>\mathrm{C}$ & $\mathrm{A} / \mathrm{A}$ & $59(50.9)$ & $4(36.4)$ & & & & \\
\hline & & $\mathrm{A} / \mathrm{C}$ & $49(42.2)$ & $6(54.5)$ & Dominant & 0.530 & 0.552 & $0.153-1.988$ \\
\hline & & $\mathrm{C} / \mathrm{C}$ & $8(6.9)$ & $1(9.1)$ & Recessive & 0.570 & 0.741 & $0.084-6.536$ \\
\hline & rs3751143 & MAF & 0.319 & 0.455 & Allele & 0.196 & 0.562 & $0.232-1.361$ \\
\hline & $\mathrm{T}>\mathrm{G}$ & $\mathrm{T} / \mathrm{T}$ & $54(46.6)$ & $2(27.3)$ & & & & \\
\hline & & $\mathrm{T} / \mathrm{G}$ & $50(43.1)$ & $6(54.5)$ & Dominant & 0.343 & 0.431 & $0.109-1.705$ \\
\hline & & $\mathrm{G} / \mathrm{G}$ & $12(10.3)$ & $2(18.2)$ & Recessive & 0.348 & 0.519 & $0.100-2.689$ \\
\hline CARD 8 & rs1971783 & MAF & 0.478 & 0.455 & Allele & 0.830 & 1.101 & $0.458-2.648$ \\
\hline & $\mathrm{T}>\mathrm{C}$ & $\mathrm{T} / \mathrm{T}$ & $28(24.1)$ & $3(27.3)$ & & & & \\
\hline & & $\mathrm{T} / \mathrm{C}$ & $65(56.0)$ & $6(54.5)$ & Dominant & 0.729 & 1.179 & $0.293-4.748$ \\
\hline & & $\mathrm{C} / \mathrm{C}$ & $23(19.8)$ & $2(18.2)$ & Recessive & 1.000 & 1.113 & $0.225-5.507$ \\
\hline & rs4389238 & MAF & 0.297 & 0.182 & Allele & 0.328 & 1.905 & $0.622-5.834$ \\
\hline & $\mathrm{C}>\mathrm{T}$ & $\mathrm{C} / \mathrm{C}$ & $56(48.3)$ & $7(63.6)$ & & & & \\
\hline & & $\mathrm{C} / \mathrm{T}$ & $51(44.0)$ & $4(36.4)$ & Dominant & 0.364 & 1.875 & $0.521-6.752$ \\
\hline & & $\mathrm{T} / \mathrm{T}$ & $9(7.8)$ & $0(0)$ & Recessive & 1.000 & 2.033 & $0.111-37.03$ \\
\hline & rs 4802448 & MAF & 0.310 & 0.227 & Allele & 0.478 & 1.530 & $0.543-4.308$ \\
\hline & $\mathrm{G}>\mathrm{A}$ & $\mathrm{G} / \mathrm{G}$ & $58(50.0)$ & $7(63.6)$ & & & & \\
\hline & & $\mathrm{G} / \mathrm{A}$ & $44(37.9)$ & $3(27.3)$ & Dominant & 0.531 & 1.750 & $0.486-6.301$ \\
\hline & & $\mathrm{A} / \mathrm{A}$ & $14(12.1)$ & $1(9.1)$ & Recessive & 1.000 & 1.372 & $0.163-11.55$ \\
\hline & rs 4802449 & MAF & 0.220 & 0.455 & Allele & 0.014 & 0.338 & $0.138-0.827$ \\
\hline & $\mathrm{G}>\mathrm{A}$ & $\mathrm{G} / \mathrm{G}$ & $70(60.3)$ & $3(27.3)$ & & & & \\
\hline & & $\mathrm{G} / \mathrm{A}$ & $41(35.3)$ & $6(54.5)$ & Dominant & 0.053 & 0.246 & $0.062-0.978$ \\
\hline & & $\mathrm{A} / \mathrm{A}$ & $5(4.3)$ & $2(18.2)$ & Recessive & 0.113 & 0.203 & $0.034-1.196$ \\
\hline & rs10418189 & MAF & 0.474 & 0.318 & Allele & 0.161 & 1.932 & $0.760-4.914$ \\
\hline & $\mathrm{G}>\mathrm{A}$ & $\mathrm{G} / \mathrm{G}$ & $31(26.7)$ & $5(45.5)$ & & & & \\
\hline & & $\mathrm{G} / \mathrm{A}$ & $60(51.7)$ & $5(45.5)$ & Dominant & 0.291 & 2.285 & $0.651-8.026$ \\
\hline & & $\mathrm{A} / \mathrm{A}$ & $25(21.6)$ & $1(9.1)$ & Recessive & 0.459 & 2.747 & $0.335-22.47$ \\
\hline & rs16981845 & MAF & 0.034 & 0.136 & Allele & 0.001 & 0.032 & $0.007-0.130$ \\
\hline & $\mathrm{T}>\mathrm{C}$ & $\mathrm{T} / \mathrm{T}$ & $108(93.1)$ & $8(72.7)$ & & & & \\
\hline & & $\mathrm{T} / \mathrm{C}$ & $8(6.9)$ & $3(27.3)$ & Dominant & 0.055 & 0.198 & $0.044-0.894$ \\
\hline & & $\mathrm{C} / \mathrm{C}$ & $0(0)$ & $0(0)$ & Recessive & - & - & - \\
\hline & rs 11670259 & MAF & 0.172 & 0.364 & Allele & 0.029 & 0.365 & $0.143-0.927$ \\
\hline & $\mathrm{C}>\mathrm{T}$ & $\mathrm{C} / \mathrm{C}$ & $79(68.1)$ & $4(36.4)$ & & & & \\
\hline & & $\mathrm{C} / \mathrm{T}$ & $34(29.3)$ & $6(54.5)$ & Dominant & 0.047 & 0.268 & $0.074-0.971$ \\
\hline & & $\mathrm{T} / \mathrm{T}$ & $3(2.6)$ & $1(9.1)$ & Recessive & 0.307 & 0.265 & $0.025-2.794$ \\
\hline & rs 12984929 & MAF & 0.349 & 0.500 & Allele & 0.159 & 0.536 & $0.223-1.291$ \\
\hline & $\mathrm{G}>\mathrm{T}$ & $\mathrm{G} / \mathrm{G}$ & $52(44.8)$ & $2(18.2)$ & & & & \\
\hline & & $\mathrm{G} / \mathrm{T}$ & $47(40.5)$ & $7(63.6)$ & Dominant & 0.115 & 0.274 & $0.057-1.322$ \\
\hline & & $\mathrm{T} / \mathrm{T}$ & $17(14.7)$ & $2(18.2)$ & Recessive & 0.669 & 0.773 & $0.153-3.890$ \\
\hline & rs11672725 & MAF & 0.190 & 0.318 & Allele & 0.150 & 0.502 & $0.193-1.304$ \\
\hline & $\mathrm{C}>\mathrm{T}$ & $\mathrm{C} / \mathrm{C}$ & $74(63.8)$ & $5(45.5)$ & & & & \\
\hline & & $\mathrm{C} / \mathrm{T}$ & $40(34.5)$ & $5(45.5)$ & Dominant & 0.330 & 0.473 & $0.136-1.644$ \\
\hline & & $\mathrm{T} / \mathrm{T}$ & $2(1.7)$ & $1(9.1)$ & Recessive & 0.240 & 0.175 & $0.015-2.107$ \\
\hline & rs6509368 & MAF & 0.388 & 0.273 & Allele & 0.287 & 1.690 & $0.638-4.480$ \\
\hline & $\mathrm{G}>\mathrm{A}$ & $\mathrm{G} / \mathrm{G}$ & $47(40.5)$ & $5(45.5)$ & & & & \\
\hline & & $\mathrm{G} / \mathrm{A}$ & $48(41.4)$ & $6(54.5)$ & Dominant & 0.758 & 1.223 & $0.353-4.243$ \\
\hline
\end{tabular}


Araki C (2016) The evidence of genetic polymorphisms of genes involved in the $P 2 R X 7$ signaling pathway as predictive biomarkers for response and loss of response to infliximab against Crohn's disease

\begin{tabular}{|c|c|c|c|c|c|c|c|c|}
\hline & & $\mathrm{A} / \mathrm{A}$ & $21(18.1)$ & $0(0)$ & Recessive & 0.209 & 5.178 & $0.293-91.37$ \\
\hline \multirow[t]{4}{*}{ PYCARD } & rs8056505 & MAF & 0.159 & 0.136 & Allele & 1.000 & 1.202 & $0.338-4.268$ \\
\hline & $\mathrm{T}>\mathrm{C}$ & $\mathrm{T} / \mathrm{T}$ & $82(70.2)$ & $8(72.7)$ & & & & \\
\hline & & $\mathrm{T} / \mathrm{C}$ & $31(26.7)$ & $3(27.3)$ & Dominant & 1.000 & 1.106 & $0.277-4.421$ \\
\hline & & $\mathrm{C} / \mathrm{C}$ & $3(2.6)$ & $0(0)$ & Recessive & 1.000 & 0.709 & $0.034-14.61$ \\
\hline \multirow[t]{4}{*}{$C A S P I$} & rs2282659 & MAF & 0.273 & 0.364 & Allele & 0.358 & 0.652 & $0.261-1.630$ \\
\hline & $\mathrm{A}>\mathrm{G}$ & $\mathrm{A} / \mathrm{A}$ & $59(50.9)$ & $3(27.3)$ & & & & \\
\hline & & $\mathrm{A} / \mathrm{G}$ & $51(44.0)$ & $8(72.7)$ & Dominant & 0.207 & 0.362 & $0.092-1.434$ \\
\hline & & $\mathrm{G} / \mathrm{G}$ & $6(5.2)$ & $0(0)$ & Recessive & 1.000 & 1.353 & $0.070-25.61$ \\
\hline \multirow[t]{16}{*}{ IL18 } & rs5744247 & MAF & 0.440 & 0.364 & Allele & 0.492 & 1.373 & $0.555-3.399$ \\
\hline & $\mathrm{C}>\mathrm{G}$ & $\mathrm{C} / \mathrm{C}$ & $34(29.3)$ & $6(54.5)$ & & & & \\
\hline & & $\mathrm{C} / \mathrm{G}$ & $62(53.4)$ & $2(18.2)$ & Dominant & 0.099 & 2.894 & $0.827-10.12$ \\
\hline & & $\mathrm{G} / \mathrm{G}$ & $20(17.2)$ & $3(27.3)$ & Recessive & 0.418 & 0.556 & $0.135-2.279$ \\
\hline & rs2043055 & MAF & 0.414 & 0.364 & Allele & 0.648 & 1.235 & $0.499-3.060$ \\
\hline & $\mathrm{A}>\mathrm{G}$ & $\mathrm{A} / \mathrm{A}$ & 39 (33.6) & $5(45.5)$ & & & & \\
\hline & & $\mathrm{A} / \mathrm{G}$ & $58(50.0)$ & $4(36.4)$ & Dominant & 0.512 & 1.645 & $0.472-5.731$ \\
\hline & & $\mathrm{G} / \mathrm{G}$ & $19(16.4)$ & $2(18.2)$ & Recessive & 1.000 & 0.881 & $0.176-4.405$ \\
\hline & rs 7106524 & MAF & 0.414 & 0.364 & Allele & 0.648 & 1.235 & $0.499-3.060$ \\
\hline & $\mathrm{G}>\mathrm{A}$ & $\mathrm{G} / \mathrm{G}$ & $38(32.8)$ & $4(36.4)$ & & & & \\
\hline & & $\mathrm{G} / \mathrm{A}$ & $60(51.7)$ & $6(54.5)$ & Dominant & 0.752 & 1.173 & $0.323-4.254$ \\
\hline & & $\mathrm{A} / \mathrm{A}$ & $18(15.5)$ & $1(9.1)$ & Recessive & 1.000 & 1.837 & $0.221-15.24$ \\
\hline & rs360717 & MAF & 0.121 & 0.227 & Allele & 0.179 & 0.467 & $0.160-1.364$ \\
\hline & $\mathrm{C}>\mathrm{G}$ & $\mathrm{C} / \mathrm{C}$ & $89(76.7)$ & $6(54.5)$ & & & & \\
\hline & & $\mathrm{C} / \mathrm{G}$ & $26(22.4)$ & $5(45.5)$ & Dominant & 0.143 & 0.364 & $0.103-1.287$ \\
\hline & & $\mathrm{G} / \mathrm{G}$ & $1(0.9)$ & $0(0)$ & Recessive & 1.000 & 0.299 & $0.011-7.768$ \\
\hline \multirow[t]{24}{*}{$I L 1 B$} & rs1143643 & MAF & 0.422 & 0.545 & Allele & 0.266 & 0.609 & $0.253-1.468$ \\
\hline & $\mathrm{G}>\mathrm{A}$ & $\mathrm{G} / \mathrm{G}$ & $38(32.8)$ & $1(9.1)$ & & & & \\
\hline & & $\mathrm{G} / \mathrm{A}$ & $58(50.0)$ & $8(72.7)$ & Dominant & 0.171 & 0.205 & $0.025-1.663$ \\
\hline & & $\mathrm{A} / \mathrm{A}$ & $20(17.2)$ & $2(18.2)$ & Recessive & 1.000 & 0.937 & $0.188-4.673$ \\
\hline & rs1143633 & MAF & 0.405 & 0.545 & Allele & 0.202 & 0.568 & $0.236-1.367$ \\
\hline & $\mathrm{A}>\mathrm{G}$ & $\mathrm{A} / \mathrm{A}$ & $38(32.8)$ & $2(18.2)$ & & & & \\
\hline & & $\mathrm{A} / \mathrm{G}$ & $62(53.4)$ & $6(54.5)$ & Dominant & 0.500 & 0.456 & $0.094-2.216$ \\
\hline & & $\mathrm{G} / \mathrm{G}$ & $16(13.8)$ & $3(27.3)$ & Recessive & 0.213 & 0.427 & $0.102-1.779$ \\
\hline & rs3136558 & MAF & 0.491 & 0.364 & Allele & 0.252 & 1.691 & $0.683-4.184$ \\
\hline & $\mathrm{T}>\mathrm{C}$ & $\mathrm{T} / \mathrm{T}$ & $27(23.3)$ & $4(36.4)$ & & & & \\
\hline & & $\mathrm{T} / \mathrm{C}$ & $64(55.2)$ & $6(54.5)$ & Dominant & 0.461 & 1.884 & $0.512-6.925$ \\
\hline & & $\mathrm{C} / \mathrm{C}$ & $25(21.6)$ & $1(9.1)$ & Recessive & 0.459 & 2.747 & $0.335-22.47$ \\
\hline & rs 1143630 & MAF & 0.151 & 0.273 & Allele & 0.138 & 0.474 & $0.173-1.294$ \\
\hline & $\mathrm{C}>\mathrm{A}$ & $\mathrm{C} / \mathrm{C}$ & $84(72.4)$ & 7 (63.6) & & & & \\
\hline & & $\mathrm{C} / \mathrm{A}$ & $29(25.0)$ & $2(18.2)$ & Dominant & 0.505 & 0.667 & $0.183-2.432$ \\
\hline & & $\mathrm{A} / \mathrm{A}$ & $3(2.6)$ & $2(18.2)$ & Recessive & 0.059 & 0.119 & $0.018-0.810$ \\
\hline & rs16944 & MAF & 0.427 & 0.682 & Allele & 0.022 & 0.347 & $0.136-0.884$ \\
\hline & $\mathrm{G}>\mathrm{A}$ & $\mathrm{G} / \mathrm{G}$ & $38(32.8)$ & $0(0)$ & & & & \\
\hline & & $\mathrm{G} / \mathrm{A}$ & $57(49.1)$ & 7 (63.6) & Dominant & 0.033 & 0.089 & $0.005-1.545$ \\
\hline & & $\mathrm{A} / \mathrm{A}$ & $21(18.1)$ & $4(36.4)$ & Recessive & 0.225 & 0.387 & $0.104-1.443$ \\
\hline & rs1143623 & MAF & 0.336 & 0.636 & Allele & 0.005 & 0.289 & $0.116-0.719$ \\
\hline & $\mathrm{G}>\mathrm{C}$ & $\mathrm{G} / \mathrm{G}$ & $52(44.8)$ & $1(9.1)$ & & & & \\
\hline & & $\mathrm{G} / \mathrm{C}$ & $50(43.1)$ & $6(54.5)$ & Dominant & 0.025 & 0.123 & $0.015-0.993$ \\
\hline & & $\mathrm{C} / \mathrm{C}$ & $14(12.1)$ & $4(36.4)$ & Recessive & 0.050 & 0.240 & $0.062-0.926$ \\
\hline
\end{tabular}

*Allele: allele model; Dominant: the minor allele dominant model; Recessive: the minor allele recessive model.( IFX, infliximab; CD, Crohn's disease; SNP, single nucleotide polymorphism; OR, odds ratio; CI, confidence interval; MAF, minor allele frequency).

There were no significant differences in the frequencies of any other alleles and genotypes at tag SNPs between responders and nonresponders after 1 year of treatment.

\section{Association of tag SNPs with response to IFX after 2 years of treatment}

The frequencies and distributions of minor alleles and genotypes at tag SNPs in each gene were identified and compared between responders and non-responders to IFX after 2 years of treatment (Table 5).
No significant differences in the frequencies of alleles and genotypes at tag SNPs were observed between responders and non-responders after 2 years of treatment (Table 5).

The interaction of genetic and environmental factors in response to IFX after 10 weeks of treatment

Univariate analyses of the differences in the frequencies of the genotypes between responders and non-responders indicated that genetic factors, the $\mathrm{C} / \mathrm{C}$ genotype of rs11670259 in CARD8 and the G/G genotype of rs 1143623 in $I L 1 B$, as well as the environmental factor 
Araki C (2016) The evidence of genetic polymorphisms of genes involved in the $P 2 R X 7$ signaling pathway as predictive biomarkers for response and loss of response to infliximab against Crohn's disease

Table 4. Allele and genotype comparisons in three inheritance models between responders and non-responders to IFX after 1 year of treatment for CD patients

\begin{tabular}{|c|c|c|c|c|c|c|c|c|}
\hline \multirow[t]{2}{*}{ Gene } & \multirow{2}{*}{$\begin{array}{c}\text { Tag SNP } \\
\text { (Major }>\text { Minor) }\end{array}$} & \multirow[t]{2}{*}{ Genotype } & \multicolumn{2}{|c|}{ Number (\%) of CD patients } & \multirow[t]{2}{*}{ Inheritance model* } & \multirow[t]{2}{*}{$P$ value } & \multirow[t]{2}{*}{ OR } & \multirow[t]{2}{*}{$95 \% \mathrm{CI}$} \\
\hline & & & $\begin{array}{l}\text { Responders } \\
(n=97)\end{array}$ & $\begin{array}{l}\text { Non-responders } \\
\quad(n=19)\end{array}$ & & & & \\
\hline \multirow[t]{44}{*}{$P 2 R X 7$} & rs 684201 & MAF & 0.222 & 0.158 & Allele & 0.379 & 1.519 & $0.596-3.870$ \\
\hline & $\mathrm{G}>\mathrm{A}$ & $\mathrm{G} / \mathrm{G}$ & $59(60.8)$ & $13(68.4)$ & & & & \\
\hline & & $\mathrm{G} / \mathrm{A}$ & $33(34.0)$ & $6(31.6)$ & Dominant & 0.533 & 1.395 & $0.488-3.987$ \\
\hline & & $\mathrm{A} / \mathrm{A}$ & $5(5.2)$ & $0(0)$ & Recessive & 0.590 & 2.319 & $0.123-43.72$ \\
\hline & rs656612 & MAF & 0.320 & 0.237 & Allele & 0.312 & 1.514 & $0.676-3.390$ \\
\hline & $\mathrm{A}>\mathrm{C}$ & $\mathrm{A} / \mathrm{A}$ & $41(42.3)$ & $11(57.9)$ & & & & \\
\hline & & $\mathrm{A} / \mathrm{C}$ & $50(51.5)$ & $7(36.8)$ & Dominant & 0.210 & 1.878 & $0.694-5.084$ \\
\hline & & $\mathrm{C} / \mathrm{C}$ & $6(6.2)$ & $1(5.3)$ & Recessive & 1.000 & 1.187 & $0.135-10.46$ \\
\hline & rs 11065450 & MAF & 0.361 & 0.184 & Allele & 0.035 & 2.500 & $1.046-5.974$ \\
\hline & $\mathrm{C}>\mathrm{A}$ & $\mathrm{C} / \mathrm{C}$ & $39(40.2)$ & $12(63.2)$ & & & & \\
\hline & & $\mathrm{C} / \mathrm{A}$ & $46(47.4)$ & $7(36.8)$ & Dominant & 0.065 & 2.550 & $0.922-7.047$ \\
\hline & & $\mathrm{A} / \mathrm{A}$ & $12(12.4)$ & $0(0)$ & Recessive & 0.211 & 5.702 & $0.323-100.6$ \\
\hline & rs1718125 & MAF & 0.273 & 0.211 & Allele & 0.422 & 1.410 & $0.608-3.270$ \\
\hline & $\mathrm{G}>\mathrm{A}$ & $\mathrm{G} / \mathrm{G}$ & $51(52.6)$ & $12(63.2)$ & & & & \\
\hline & & $\mathrm{G} / \mathrm{A}$ & $39(40.2)$ & $6(31.6)$ & Dominant & 0.397 & 1.546 & $0.561-4.261$ \\
\hline & & $\mathrm{A} / \mathrm{A}$ & $7(7.2)$ & $1(5.3)$ & Recessive & 1.000 & 1.400 & $0.162-12.09$ \\
\hline & rs1186055 & MAF & 0.392 & 0.395 & Allele & 0.973 & 0.988 & $0.485-2.012$ \\
\hline & $\mathrm{G}>\mathrm{T}$ & $\mathrm{G} / \mathrm{G}$ & $36(37.1)$ & $6(31.6)$ & & & & \\
\hline & & $\mathrm{G} / \mathrm{T}$ & $46(47.4)$ & $11(57.9)$ & Dominant & 0.646 & 0.782 & $0.273-2.238$ \\
\hline & & $\mathrm{T} / \mathrm{T}$ & $15(15.5)$ & $2(10.5)$ & Recessive & 0.735 & 1.555 & $0.325-7.435$ \\
\hline & rs208296 & MAF & 0.340 & 0.211 & Allele & 0.117 & 1.933 & $0.839-4.454$ \\
\hline & $\mathrm{C}>\mathrm{T}$ & $\mathrm{C} / \mathrm{C}$ & $40(41.2)$ & $11(57.9)$ & & & & \\
\hline & & $\mathrm{C} / \mathrm{T}$ & $48(49.5)$ & $8(42.1)$ & Dominant & 0.181 & 1.959 & $0.723-5.308$ \\
\hline & & $\mathrm{T} / \mathrm{T}$ & $9(9.3)$ & $0(0)$ & Recessive & 0.352 & 4.186 & $0.234-75.06$ \\
\hline & rs 11065464 & MAF & 0.247 & 0.184 & Allele & 0.402 & 1.456 & $0.602-3.520$ \\
\hline & $\mathrm{C}>\mathrm{A}$ & $\mathrm{C} / \mathrm{C}$ & $54(55.7)$ & $13(68.4)$ & & & & \\
\hline & & $\mathrm{C} / \mathrm{A}$ & $38(39.2)$ & $5(26.3)$ & Dominant & 0.304 & 1.725 & $0.606-4.916$ \\
\hline & & $\mathrm{A} / \mathrm{A}$ & $5(5.2)$ & $1(5.3)$ & Recessive & 1.000 & 0.978 & $0.108-8.881$ \\
\hline & rs208307 & MAF & 0.155 & 0.105 & Allele & 0.616 & 1.555 & $0.514-4.704$ \\
\hline & $\mathrm{G}>\mathrm{C}$ & $\mathrm{G} / \mathrm{G}$ & $70(72.2)$ & $16(84.2)$ & & & & \\
\hline & & $\mathrm{G} / \mathrm{C}$ & $24(24.7)$ & $2(10.5)$ & Dominant & 0.393 & 2.057 & $0.555-7.628$ \\
\hline & & $\mathrm{C} / \mathrm{C}$ & $3(3.1)$ & $1(5.3)$ & Recessive & 0.516 & 0.574 & $0.057-5.838$ \\
\hline & rs7958311 & MAF & 0.361 & 0.289 & Allele & 0.399 & 1.386 & $0.648-2.962$ \\
\hline & $\mathrm{G}>\mathrm{A}$ & $\mathrm{G} / \mathrm{G}$ & $41(42.3)$ & $12(63.2)$ & & & & \\
\hline & & $\mathrm{G} / \mathrm{A}$ & $42(43.3)$ & $3(15.8)$ & Dominant & 0.095 & 2.341 & $0.848-6.464$ \\
\hline & & $\mathrm{A} / \mathrm{A}$ & $14(14.4)$ & $4(21.1)$ & Recessive & 0.492 & 0.633 & $0.183-2.185$ \\
\hline & rs1653609 & MAF & 0.294 & 0.211 & Allele & 0.296 & 1.560 & $0.674-3.610$ \\
\hline & $\mathrm{A}>\mathrm{C}$ & $\mathrm{A} / \mathrm{A}$ & $46(47.4)$ & $13(68.4)$ & & & & \\
\hline & & $\mathrm{A} / \mathrm{C}$ & $45(46.4)$ & $4(21.1)$ & Dominant & 0.094 & 2.402 & $0.844-6.840$ \\
\hline & & $\mathrm{C} / \mathrm{C}$ & $6(6.2)$ & $2(10.5)$ & Recessive & 0.616 & 0.560 & $0.104-3.013$ \\
\hline & rs3751143 & MAF & 0.289 & 0.474 & Allele & 0.025 & 0.451 & $0.222-0.916$ \\
\hline & $\mathrm{T}>\mathrm{G}$ & $\mathrm{T} / \mathrm{T}$ & $47(48.5)$ & $7(36.8)$ & & & & \\
\hline & & $\mathrm{T} / \mathrm{G}$ & $44(45.5)$ & $6(31.6)$ & Dominant & 0.354 & 0.621 & $0.225-1.710$ \\
\hline & & $\mathrm{G} / \mathrm{G}$ & $6(6.2)$ & $6(31.6)$ & Recessive & 0.001 & 0.143 & $0.040-0.510$ \\
\hline \multirow[t]{14}{*}{ CARD 8} & rs1971783 & MAF & 0.490 & 0.421 & Allele & 0.439 & 1.319 & $0.653-2.665$ \\
\hline & $\mathrm{T}>\mathrm{C}$ & $\mathrm{T} / \mathrm{T}$ & $21(21.6)$ & $7(36.8)$ & & & & \\
\hline & & $\mathrm{T} / \mathrm{C}$ & $57(58.8)$ & $8(42.1)$ & Dominant & 0.157 & 2.111 & $0.739-6.031$ \\
\hline & & $\mathrm{C} / \mathrm{C}$ & $19(19.6)$ & $4(21.1)$ & Recessive & 1.000 & 0.913 & $0.272-3.068$ \\
\hline & rs4389238 & MAF & 0.268 & 0.447 & Allele & 0.027 & 0.452 & $0.222-0.924$ \\
\hline & $\mathrm{C}>\mathrm{T}$ & $\mathrm{C} / \mathrm{C}$ & $52(53.6)$ & $4(21.1)$ & & & & \\
\hline & & $\mathrm{C} / \mathrm{T}$ & $38(39.2)$ & $13(68.4)$ & Dominant & 0.012 & 0.231 & $0.071-0.746$ \\
\hline & & $\mathrm{T} / \mathrm{T}$ & $7(7.2)$ & $2(10.5)$ & Recessive & 0.640 & 0.661 & $0.126-3.459$ \\
\hline & rs 4802448 & MAF & 0.309 & 0.316 & Allele & 0.937 & 0.970 & $0.459-2.051$ \\
\hline & $\mathrm{G}>\mathrm{A}$ & $\mathrm{G} / \mathrm{G}$ & $48(49.5)$ & $10(52.6)$ & & & & \\
\hline & & $\mathrm{G} / \mathrm{A}$ & $38(39.2)$ & $6(31.6)$ & Dominant & 0.802 & 1.134 & $0.424-3.036$ \\
\hline & & $\mathrm{A} / \mathrm{A}$ & $11(11.3)$ & $3(15.8)$ & Recessive & 0.699 & 0.682 & $0.171-2.722$ \\
\hline & rs4802449 & MAF & 0.216 & 0.237 & Allele & 0.782 & 0.890 & $0.391-2.026$ \\
\hline & $\mathrm{G}>\mathrm{A}$ & $\mathrm{G} / \mathrm{G}$ & $60(61.9)$ & $10(52.6)$ & & & & \\
\hline
\end{tabular}


Araki C (2016) The evidence of genetic polymorphisms of genes involved in the $P 2 R X 7$ signaling pathway as predictive biomarkers for response and loss of response to infliximab against Crohn's disease

\begin{tabular}{|c|c|c|c|c|c|c|c|c|}
\hline & & $\mathrm{G} / \mathrm{A}$ & $32(33.0)$ & $9(47.4)$ & Dominant & 0.452 & 0.685 & $0.255-1.843$ \\
\hline & & $\mathrm{A} / \mathrm{A}$ & $5(5.2)$ & $0(0)$ & Recessive & 0.590 & 2.319 & $0.123-43.72$ \\
\hline & rs10418189 & MAF & 0.479 & 0.447 & Allele & 0.718 & 1.137 & $0.566-2.288$ \\
\hline & $\mathrm{G}>\mathrm{A}$ & $\mathrm{G} / \mathrm{G}$ & $25(25.8)$ & $6(31.6)$ & & & & \\
\hline & & $\mathrm{G} / \mathrm{A}$ & $51(52.6)$ & $9(47.4)$ & Dominant & 0.601 & 1.329 & $0.456-3.871$ \\
\hline & & $\mathrm{A} / \mathrm{A}$ & $21(21.6)$ & $4(21.1)$ & Recessive & 1.000 & 1.036 & $0.311-3.454$ \\
\hline & rs16981845 & MAF & 0.036 & 0.026 & Allele & 1.000 & 1.385 & $0.165-11.49$ \\
\hline & $\mathrm{T}>\mathrm{C}$ & $\mathrm{T} / \mathrm{T}$ & $90(92.8)$ & $18(94.7)$ & & & & \\
\hline & & $\mathrm{T} / \mathrm{C}$ & $7(7.2)$ & $1(5.3)$ & Dominant & 1.000 & 0.714 & $0.083-6.169$ \\
\hline & & $\mathrm{C} / \mathrm{C}$ & $0(0)$ & $0(0)$ & Recessive & - & - & - \\
\hline & rs 11670259 & MAF & 0.170 & 0.184 & Allele & 0.833 & 0.908 & $0.368-2.236$ \\
\hline & $\mathrm{C}>\mathrm{T}$ & $\mathrm{C} / \mathrm{C}$ & $67(69.1)$ & $12(63.2)$ & & & & \\
\hline & & $\mathrm{C} / \mathrm{T}$ & $27(27.8)$ & $7(36.8)$ & Dominant & 0.613 & 0.768 & $0.275-2.143$ \\
\hline & & $\mathrm{T} / \mathrm{T}$ & $3(3.1)$ & $0(0)$ & Recessive & 1.000 & 1.444 & $0.071-29.41$ \\
\hline & rs12984929 & MAF & 0.351 & 0.342 & Allele & 0.921 & 1.038 & $0.499-2.158$ \\
\hline & $\mathrm{G}>\mathrm{T}$ & $\mathrm{G} / \mathrm{G}$ & $44(45.4)$ & $8(42.1)$ & & & & \\
\hline & & $\mathrm{G} / \mathrm{T}$ & $38(39.2)$ & $9(47.4)$ & Dominant & 0.794 & 0.876 & $0.324-2.369$ \\
\hline & & $\mathrm{T} / \mathrm{T}$ & $15(15.5)$ & $2(10.5)$ & Recessive & 0.735 & 1.555 & $0.325-7.435$ \\
\hline & rs11672725 & MAF & 0.186 & 0.211 & Allele & 0.720 & 0.854 & $0.362-2.019$ \\
\hline & $\mathrm{C}>\mathrm{T}$ & $\mathrm{C} / \mathrm{C}$ & $63(64.9)$ & $11(57.9)$ & & & & \\
\hline & & $\mathrm{C} / \mathrm{T}$ & $32(33.0)$ & $8(42.1)$ & Dominant & 0.559 & 0.742 & $0.273-2.021$ \\
\hline & & $\mathrm{T} / \mathrm{T}$ & $2(2.1)$ & $0(0)$ & Recessive & 1.000 & 1.021 & $0.047-22.22$ \\
\hline & rs6509368 & MAF & 0.376 & 0.447 & Allele & 0.411 & 0.745 & $0.369-1.504$ \\
\hline & $\mathrm{G}>\mathrm{A}$ & $\mathrm{G} / \mathrm{G}$ & $41(42.3)$ & $6(31.6)$ & & & & \\
\hline & & $\mathrm{G} / \mathrm{A}$ & $49(40.2)$ & $9(47.4)$ & Dominant & 0.386 & 0.630 & $0.221-1.798$ \\
\hline & & $\mathrm{A} / \mathrm{A}$ & $17(17.5)$ & $4(21.1)$ & Recessive & 0.747 & 0.797 & $0.235-2.701$ \\
\hline PYCARD & rs8056505 & MAF & 0.165 & 0.132 & Allele & 0.809 & 1.304 & $0.473-3.595$ \\
\hline & $\mathrm{T}>\mathrm{C}$ & $\mathrm{T} / \mathrm{T}$ & $68(70.1)$ & $14(73.7)$ & & & & \\
\hline & & $\mathrm{T} / \mathrm{C}$ & $26(26.8)$ & $5(26.3)$ & Dominant & 1.000 & 1.194 & $0.394-3.623$ \\
\hline & & $\mathrm{C} / \mathrm{C}$ & $3(3.1)$ & $0(0)$ & Recessive & 1.000 & 1.444 & $0.072-29.12$ \\
\hline CASPI & rs2282659 & MAF & 0.309 & 0.079 & Allele & 0.003 & 5.225 & $1.546-17.67$ \\
\hline & $A>G$ & $\mathrm{~A} / \mathrm{A}$ & $43(44.3)$ & $16(84.2)$ & & & & \\
\hline & & $\mathrm{A} / \mathrm{G}$ & $48(49.5)$ & $3(15.8)$ & Dominant & 0.002 & 6.698 & $1.832-24.51$ \\
\hline & & $\mathrm{G} / \mathrm{G}$ & $6(6.2)$ & $0(0)$ & Recessive & 0.587 & 2.770 & $0.150-51.29$ \\
\hline IL18 & rs5744247 & MAF & 0.443 & 0.421 & Allele & 0.801 & 1.095 & $0.542-2.213$ \\
\hline & $\mathrm{C}>\mathrm{G}$ & $\mathrm{C} / \mathrm{C}$ & $29(29.9)$ & $5(26.3)$ & & & & \\
\hline & & $\mathrm{C} / \mathrm{G}$ & $50(51.5)$ & $12(63.2)$ & Dominant & 1.000 & 0.837 & $0.276-2.541$ \\
\hline & & $\mathrm{G} / \mathrm{G}$ & $18(18.6)$ & $2(10.5)$ & Recessive & 0.521 & 1.937 & $0.410-9.141$ \\
\hline & rs2043055 & MAF & 0.407 & 0.447 & Allele & 0.646 & 0.849 & $0.421-1.710$ \\
\hline & $\mathrm{A}>\mathrm{G}$ & $\mathrm{A} / \mathrm{A}$ & $35(36.1)$ & $4(21.1)$ & & & & \\
\hline & & $\mathrm{A} / \mathrm{G}$ & $45(46.4)$ & $13(68.4)$ & Dominant & 0.290 & 0.472 & $0.145-1.535$ \\
\hline & & $\mathrm{G} / \mathrm{G}$ & $17(17.5)$ & $2(10.5)$ & Recessive & 0.735 & 1.806 & $0.381-8.562$ \\
\hline & rs7106524 & MAF & 0.402 & 0.474 & Allele & 0.412 & 0.747 & $0.372-1.502$ \\
\hline & $\mathrm{G}>\mathrm{A}$ & $\mathrm{G} / \mathrm{G}$ & $34(35.1)$ & $4(21.1)$ & & & & \\
\hline & & $\mathrm{G} / \mathrm{A}$ & $48(49.1)$ & $12(63.2)$ & Dominant & 0.293 & 0.494 & $0.152-1.607$ \\
\hline & & $\mathrm{A} / \mathrm{A}$ & $15(15.5)$ & $3(15.8)$ & Recessive & 1.000 & 0.976 & $0.253-3.765$ \\
\hline & rs360717 & MAF & 0.119 & 0.132 & Allele & 0.788 & 0.888 & $0.315-2.503$ \\
\hline & $\mathrm{C}>\mathrm{G}$ & $\mathrm{C} / \mathrm{C}$ & $75(77.3)$ & $14(73.7)$ & & & & \\
\hline & & $\mathrm{C} / \mathrm{G}$ & $21(21.6)$ & $5(26.3)$ & Dominant & 0.769 & 0.821 & $0.266-2.533$ \\
\hline & & $\mathrm{G} / \mathrm{G}$ & $1(1.0)$ & $0(0)$ & Recessive & 1.000 & 0.606 & $0.024-15.45$ \\
\hline$I L 1 B$ & rs1143643 & MAF & 0.428 & 0.395 & Allele & 0.706 & 1.147 & $0.564-2.331$ \\
\hline & $\mathrm{G}>\mathrm{A}$ & $\mathrm{G} / \mathrm{G}$ & $31(32.0)$ & $7(36.8)$ & & & & \\
\hline & & $\mathrm{G} / \mathrm{A}$ & $49(50.5)$ & $9(47.4)$ & Dominant & 0.678 & 1.242 & $0.446-3.463$ \\
\hline & & $\mathrm{A} / \mathrm{A}$ & $17(17.5)$ & $3(15.8)$ & Recessive & 1.000 & 1.133 & $0.297-4.327$ \\
\hline & rs1143633 & MAF & 0.421 & 0.368 & Allele & 0.614 & 1.203 & $0.586-2.468$ \\
\hline & $A>G$ & $\mathrm{~A} / \mathrm{A}$ & $31(32.0)$ & $7(36.8)$ & & & & \\
\hline & & $\mathrm{A} / \mathrm{G}$ & $52(53.6)$ & $10(52.6)$ & Dominant & 0.678 & 1.242 & $0.446-3.463$ \\
\hline & & $\mathrm{G} / \mathrm{G}$ & $14(14.4)$ & $2(10.5)$ & Recessive & 1.000 & 1.434 & $0.298-6.897$ \\
\hline & rs3136558 & MAF & 0.469 & 0.605 & Allele & 0.125 & 0.576 & $0.284-1.171$ \\
\hline & $\mathrm{T}>\mathrm{C}$ & $\mathrm{T} / \mathrm{T}$ & $25(25.8)$ & $2(10.5)$ & & & & \\
\hline & & $\mathrm{T} / \mathrm{C}$ & $53(54.6)$ & $11(57.9)$ & Dominant & 0.235 & 0.339 & $0.073-1.571$ \\
\hline & & $\mathrm{C} / \mathrm{C}$ & $19(19.6)$ & $6(31.6)$ & Recessive & 0.245 & 0.528 & $0.178-1.569$ \\
\hline
\end{tabular}


Araki C (2016) The evidence of genetic polymorphisms of genes involved in the $P 2 R X 7$ signaling pathway as predictive biomarkers for response and loss of response to infliximab against Crohn's disease

\begin{tabular}{|c|c|c|c|c|c|c|c|}
\hline rs1143630 & MAF & 0.144 & 0.184 & Allele & 0.530 & 0.747 & $0.300-1.861$ \\
\hline $\mathrm{C}>\mathrm{A}$ & $\mathrm{C} / \mathrm{C}$ & $72(74.2)$ & $12(63.2)$ & & & & \\
\hline & $\mathrm{C} / \mathrm{A}$ & $22(22.7)$ & $7(36.8)$ & Dominant & 0.324 & 0.595 & $0.211-1.680$ \\
\hline & $\mathrm{A} / \mathrm{A}$ & $3(3.1)$ & $0(0)$ & Recessive & 1.000 & 1.444 & $0.072-29.12$ \\
\hline rs16944 & MAF & 0.448 & 0.316 & Allele & 0.131 & 1.762 & $0.840-3.693$ \\
\hline $\mathrm{G}>\mathrm{A}$ & $\mathrm{G} / \mathrm{G}$ & $30(30.9)$ & $8(42.1)$ & & & & \\
\hline & $\mathrm{G} / \mathrm{A}$ & $47(48.5)$ & $10(52.6)$ & Dominant & 0.343 & 1.624 & $0.593-4.448$ \\
\hline & $\mathrm{A} / \mathrm{A}$ & $20(20.6)$ & $1(5.3)$ & Recessive & 0.190 & 4.675 & $0.588-37.18$ \\
\hline rs1143623 & MAF & 0.351 & 0.263 & Allele & 0.297 & 1.511 & $0.693-3.296$ \\
\hline $\mathrm{G}>\mathrm{C}$ & $\mathrm{G} / \mathrm{G}$ & $42(43.3)$ & $10(52.6)$ & & & & \\
\hline & $\mathrm{G} / \mathrm{C}$ & $42(43.3)$ & $8(42.1)$ & Dominant & 0.455 & 1.455 & $0.543-3.900$ \\
\hline & $\mathrm{C} / \mathrm{C}$ & $13(13.4)$ & $1(5.3)$ & Recessive & 0.461 & 2.786 & $0.342-22.68$ \\
\hline
\end{tabular}

* Allele: allele model; Dominant: the minor allele dominant model; Recessive: the minor allele recessive model. (IFX, infliximab; CD, Crohn's disease; SNP, single nucleotide polymorphism; OR, odds ratio; CI, confidence interval; MAF, minor allele frequency).

Table 5. Allele and genotype comparisons in three inheritance models between responders and non-responders to IFX after 2 years of treatment for CD patients.

\begin{tabular}{|c|c|c|c|c|c|c|c|c|}
\hline \multirow[t]{2}{*}{ Gene } & \multirow{2}{*}{$\begin{array}{c}\text { Tag SNP } \\
\text { (Major }>\text { Minor })\end{array}$} & \multirow[t]{2}{*}{ Genotype } & \multicolumn{2}{|c|}{ Number (\%) of CD patients } & \multirow[t]{2}{*}{ Inheritance model* } & \multirow[t]{2}{*}{$P$ value } & \multirow[t]{2}{*}{ OR } & \multirow[t]{2}{*}{$95 \%$ CI } \\
\hline & & & $\begin{array}{l}\text { Responders } \\
\quad(n=82)\end{array}$ & $\begin{array}{l}\text { Non-responders } \\
\quad(n=15)\end{array}$ & & & & \\
\hline \multirow[t]{42}{*}{$P 2 R X 7$} & rs684201 & MAF & 0.244 & 0.100 & Allele & 0.096 & 2.904 & $0.836-10.08$ \\
\hline & $\mathrm{G}>\mathrm{A}$ & $\mathrm{G} / \mathrm{G}$ & $47(57.3)$ & $12(80.0)$ & & & & \\
\hline & & $\mathrm{G} / \mathrm{A}$ & $30(36.6)$ & $3(20.0)$ & Dominant & 0.150 & 2.979 & $0.781-11.36$ \\
\hline & & $\mathrm{A} / \mathrm{A}$ & $5(6.1)$ & $0(0)$ & Recessive & 1.000 & 2.200 & $0.116-41.89$ \\
\hline & rs656612 & MAF & 0.317 & 0.333 & Allele & 0.861 & 0.929 & $0.406-2.124$ \\
\hline & $\mathrm{A}>\mathrm{C}$ & $\mathrm{A} / \mathrm{A}$ & $36(43.9)$ & $5(33.3)$ & & & & \\
\hline & & $\mathrm{A} / \mathrm{C}$ & $40(48.8)$ & $10(66.7)$ & Dominant & 0.574 & 0.639 & $0.201-2.035$ \\
\hline & & $\mathrm{C} / \mathrm{C}$ & $6(7.3)$ & $0(0)$ & Recessive & 0.586 & 2.634 & $0.141-49.26$ \\
\hline & rs 11065450 & MAF & 0.372 & 0.300 & Allele & 0.451 & 1.382 & $0.595-3.209$ \\
\hline & $\mathrm{C}>\mathrm{A}$ & $\mathrm{C} / \mathrm{C}$ & $33(40.2)$ & $6(40.0)$ & & & & \\
\hline & & $\mathrm{C} / \mathrm{A}$ & $37(45.1)$ & $9(60.0)$ & Dominant & 0.986 & 0.990 & $0.322-3.044$ \\
\hline & & $\mathrm{A} / \mathrm{A}$ & $12(14.6)$ & $0(0)$ & Recessive & 0.203 & 5.496 & $0.308-97.95$ \\
\hline & rs 1718125 & MAF & 0.299 & 0.133 & Allele & 0.075 & 2.769 & $0.918-8.361$ \\
\hline & $\mathrm{G}>\mathrm{A}$ & $\mathrm{G} / \mathrm{G}$ & $40(48.8)$ & $11(73.3)$ & & & & \\
\hline & & $\mathrm{G} / \mathrm{A}$ & $35(42.7)$ & $4(26.7)$ & Dominant & 0.097 & 2.888 & $0.849-9.814$ \\
\hline & & $\mathrm{A} / \mathrm{A}$ & $7(8.5)$ & $0(0)$ & Recessive & 0.591 & 3.079 & $0.167-56.82$ \\
\hline & rs1186055 & MAF & 0.396 & 0.367 & Allele & 0.760 & 1.134 & $0.507-2.539$ \\
\hline & $\mathrm{G}>\mathrm{T}$ & $\mathrm{G} / \mathrm{G}$ & $31(37.8)$ & $5(33.3)$ & & & & \\
\hline & & $\mathrm{G} / \mathrm{T}$ & $37(45.1)$ & $9(60.0)$ & Dominant & 1.000 & 0.823 & $0.257-2.631$ \\
\hline & & $\mathrm{T} / \mathrm{T}$ & $14(17.1)$ & $1(6.7)$ & Recessive & 0.454 & 2.883 & $0.350-23.75$ \\
\hline & rs208296 & MAF & 0.335 & 0.367 & Allele & 0.739 & 0.872 & $0.388-1.960$ \\
\hline & $\mathrm{C}>\mathrm{T}$ & $\mathrm{C} / \mathrm{C}$ & $35(42.7)$ & $5(33.3)$ & & & & \\
\hline & & $\mathrm{C} / \mathrm{T}$ & $39(47.6)$ & $9(60.0)$ & Dominant & 0.578 & 0.671 & $0.211-2.140$ \\
\hline & & $\mathrm{T} / \mathrm{T}$ & $8(9.8)$ & $1(6.7)$ & Recessive & 1.000 & 1.514 & $0.175-13.07$ \\
\hline & rs11065464 & MAF & 0.256 & 0.200 & Allele & 0.513 & 1.377 & $0.527-3.600$ \\
\hline & $\mathrm{C}>\mathrm{A}$ & $\mathrm{C} / \mathrm{C}$ & $44(53.7)$ & $10(66.7)$ & & & & \\
\hline & & $\mathrm{C} / \mathrm{A}$ & $34(41.5)$ & $4(26.7)$ & Dominant & 0.408 & 1.727 & $0.543-5.498$ \\
\hline & & $\mathrm{A} / \mathrm{A}$ & $4(4.9)$ & $1(6.7)$ & Recessive & 0.577 & 0.718 & $0.075-6.906$ \\
\hline & rs208307 & MAF & 0.140 & 0.233 & Allele & 0.195 & 0.536 & $0.206-1.391$ \\
\hline & $\mathrm{G}>\mathrm{C}$ & $\mathrm{G} / \mathrm{G}$ & $62(75.6)$ & $8(53.3)$ & & & & \\
\hline & & $\mathrm{G} / \mathrm{C}$ & $17(20.7)$ & $7(46.7)$ & Dominant & 0.077 & 0.369 & $0.119-1.144$ \\
\hline & & $\mathrm{C} / \mathrm{C}$ & $3(3.7)$ & $0(0)$ & Recessive & 1.000 & 1.365 & $0.067-27.78$ \\
\hline & rs7958311 & MAF & 0.366 & 0.333 & Allele & 0.733 & 1.154 & $0.507-2.627$ \\
\hline & $\mathrm{G}>\mathrm{A}$ & $\mathrm{G} / \mathrm{G}$ & $34(41.5)$ & $7(46.7)$ & & & & \\
\hline & & $\mathrm{G} / \mathrm{A}$ & $36(43.9)$ & $6(40.0)$ & Dominant & 0.708 & 1.235 & $0.409-3.731$ \\
\hline & & $\mathrm{A} / \mathrm{A}$ & $12(14.6)$ & $2(13.3)$ & Recessive & 1.000 & 1.114 & $0.223-5.574$ \\
\hline & rs1653609 & MAF & 0.293 & 0.300 & Allele & 0.936 & 0.966 & $0.413-2.259$ \\
\hline & $\mathrm{A}>\mathrm{C}$ & $\mathrm{A} / \mathrm{A}$ & $40(48.8)$ & $6(40.0)$ & & & & \\
\hline & & $\mathrm{A} / \mathrm{C}$ & $36(43.9)$ & $9(60.0)$ & Dominant & 0.531 & 0.700 & $0.228-2.145$ \\
\hline & & $\mathrm{C} / \mathrm{C}$ & $6(7.3)$ & $0(0)$ & Recessive & 0.586 & 2.634 & $0.141-49.26$ \\
\hline & rs 3751143 & MAF & 0.262 & 0.433 & Allele & 0.057 & 0.465 & $0.209-1.036$ \\
\hline & $\mathrm{T}>\mathrm{G}$ & $\mathrm{T} / \mathrm{T}$ & $43(52.4)$ & $4(26.7)$ & & & & \\
\hline
\end{tabular}


Araki C (2016) The evidence of genetic polymorphisms of genes involved in the $P 2 R X 7$ signaling pathway as predictive biomarkers for response and loss of response to infliximab against Crohn's disease

\begin{tabular}{|c|c|c|c|c|c|c|c|c|}
\hline & & $T / G$ & $35(42.7)$ & $9(60.0)$ & Dominant & 0.092 & 0.330 & $0.097-1.121$ \\
\hline & & $\mathrm{G} / \mathrm{G}$ & $4(4.9)$ & $2(13.3)$ & Recessive & 0.232 & 0.333 & $0.055-2.009$ \\
\hline \multirow[t]{40}{*}{ CARD 8} & rs 1971783 & MAF & 0.512 & 0.367 & Allele & 0.143 & 1.814 & $0.812-4.049$ \\
\hline & $\mathrm{T}>\mathrm{C}$ & $\mathrm{T} / \mathrm{T}$ & $17(20.7)$ & $4(26.7)$ & & & & \\
\hline & & $T / C$ & $46(56.1)$ & $11(73.3)$ & Dominant & 0.733 & 1.390 & $0.393-4.916$ \\
\hline & & $\mathrm{C} / \mathrm{C}$ & $19(23.2)$ & $0(0)$ & Recessive & 0.037 & 0.105 & $0.006-1.838$ \\
\hline & rs4389238 & MAF & 0.274 & 0.233 & Allele & 0.641 & 1.243 & $0.499-3.096$ \\
\hline & $\mathrm{C}>\mathrm{T}$ & $\mathrm{C} / \mathrm{C}$ & $43(52.4)$ & $9(60.0)$ & & & & \\
\hline & & $\mathrm{C} / \mathrm{T}$ & $33(40.2)$ & $5(33.3)$ & Dominant & 0.589 & 1.361 & $0.444-4.170$ \\
\hline & & $\mathrm{T} / \mathrm{T}$ & $6(7.3)$ & $1(6.7)$ & Recessive & 1.000 & 1.105 & $0.123-9.901$ \\
\hline & rs 4802448 & MAF & 0.299 & 0.367 & Allele & 0.460 & 0.736 & $0.326-1.662$ \\
\hline & $\mathrm{G}>\mathrm{A}$ & $\mathrm{G} / \mathrm{G}$ & $41(50.0)$ & $7(46.7)$ & & & & \\
\hline & & $\mathrm{G} / \mathrm{A}$ & $33(40.2)$ & $5(33.3)$ & Dominant & 0.812 & 0.875 & $0.290-2.636$ \\
\hline & & $\mathrm{A} / \mathrm{A}$ & $8(9.8)$ & $3(20.0)$ & Recessive & 0.368 & 0.432 & $0.100-1.863$ \\
\hline & rs 4802449 & MAF & 0.201 & 0.300 & Allele & 0.227 & 0.588 & $0.246-1.402$ \\
\hline & $\mathrm{G}>\mathrm{A}$ & $\mathrm{G} / \mathrm{G}$ & $52(63.4)$ & $8(53.3)$ & & & & \\
\hline & & $\mathrm{G} / \mathrm{A}$ & $27(32.9)$ & $5(33.3)$ & Dominant & 0.460 & 0.659 & $0.217-2.000$ \\
\hline & & $\mathrm{A} / \mathrm{A}$ & $3(3.7)$ & $2(13.3)$ & Recessive & 0.170 & 0.247 & $0.038-1.622$ \\
\hline & rs10418189 & MAF & 0.506 & 0.333 & Allele & 0.082 & 2.049 & $0.904-4.647$ \\
\hline & $\mathrm{G}>\mathrm{A}$ & $\mathrm{G} / \mathrm{G}$ & $20(24.4)$ & $5(33.3)$ & & & & \\
\hline & & $\mathrm{G} / \mathrm{A}$ & $41(50.0)$ & $10(66.7)$ & Dominant & 0.525 & 1.550 & $0.473-5.074$ \\
\hline & & $\mathrm{A} / \mathrm{A}$ & $21(25.6)$ & $0(0)$ & Recessive & 0.036 & 10.840 & $0.621-189.0$ \\
\hline & rs16981845 & MAF & 0.037 & 0.033 & Allele & 1.000 & 1.101 & $0.128-9.524$ \\
\hline & $\mathrm{T}>\mathrm{C}$ & $\mathrm{T} / \mathrm{T}$ & $76(92.7)$ & $14(93.3)$ & & & & \\
\hline & & $\mathrm{T} / \mathrm{C}$ & $6(7.3)$ & $1(6.7)$ & Dominant & 1.000 & 0.905 & $0.101-8.108$ \\
\hline & & $\mathrm{C} / \mathrm{C}$ & $0(0)$ & $0(0)$ & Recessive & - & - & - \\
\hline & rs11670259 & MAF & 0.152 & 0.267 & Allele & 0.126 & 0.495 & $0.198-1.234$ \\
\hline & $\mathrm{C}>\mathrm{T}$ & $\mathrm{C} / \mathrm{C}$ & $59(72.0)$ & $8(53.3)$ & & & & \\
\hline & & $\mathrm{C} / \mathrm{T}$ & $21(25.6)$ & $6(40.0)$ & Dominant & 0.152 & 0.446 & $0.145-1.369$ \\
\hline & & $\mathrm{T} / \mathrm{T}$ & $2(2.4)$ & $1(6.7)$ & Recessive & 0.399 & 0.350 & $0.030-4.124$ \\
\hline & rs 12984929 & MAF & 0.341 & 0.400 & Allele & 0.537 & 0.778 & $0.350-1.729$ \\
\hline & $\mathrm{G}>\mathrm{T}$ & $\mathrm{G} / \mathrm{G}$ & $38(46.3)$ & $6(40.0)$ & & & & \\
\hline & & $\mathrm{G} / \mathrm{T}$ & $32(39.0)$ & $6(40.0)$ & Dominant & 0.650 & 0.772 & $0.252-2.367$ \\
\hline & & $\mathrm{T} / \mathrm{T}$ & $12(14.6)$ & $3(20.0)$ & Recessive & 0.697 & 0.686 & $0.168-2.796$ \\
\hline & rs 11672725 & MAF & 0.171 & 0.267 & Allele & 0.214 & 0.566 & $0.229-1.401$ \\
\hline & $\mathrm{C}>\mathrm{T}$ & $\mathrm{C} / \mathrm{C}$ & $55(67.1)$ & $8(53.3)$ & & & & \\
\hline & & $\mathrm{C} / \mathrm{T}$ & $26(31.7)$ & $6(40.0)$ & Dominant & 0.305 & 0.561 & $0.184-1.709$ \\
\hline & & $\mathrm{T} / \mathrm{T}$ & $1(1.2)$ & $1(6.7)$ & Recessive & 0.287 & 0.173 & $0.010-2.927$ \\
\hline & rs6509368 & MAF & 0.372 & 0.400 & Allele & 0.771 & 0.888 & $0.401-1.969$ \\
\hline & $\mathrm{G}>\mathrm{A}$ & $\mathrm{G} / \mathrm{G}$ & $35(42.7)$ & $6(40.0)$ & & & & \\
\hline & & $\mathrm{G} / \mathrm{A}$ & $33(40.2)$ & $6(40.0)$ & Dominant & 0.847 & 0.895 & $0.292-2.749$ \\
\hline & & $\mathrm{A} / \mathrm{A}$ & $14(17.1)$ & $3(20.0)$ & Recessive & 0.723 & 0.824 & $0.205-3.306$ \\
\hline \multirow[t]{4}{*}{ PYCARD } & rs8056505 & MAF & 0.177 & 0.100 & Allele & 0.424 & 1.933 & $0.549-6.807$ \\
\hline & $\mathrm{T}>\mathrm{C}$ & $\mathrm{T} / \mathrm{T}$ & $56(68.3)$ & $12(80.0)$ & & & & \\
\hline & & $\mathrm{T} / \mathrm{C}$ & $23(28.0)$ & $2(20.0)$ & Dominant & 0.542 & 1.857 & $0.482-7.148$ \\
\hline & & $\mathrm{C} / \mathrm{C}$ & $3(3.7)$ & $0(0)$ & Recessive & 1.000 & 1.365 & $0.067-27.78$ \\
\hline \multirow[t]{4}{*}{$C A S P 1$} & rs2282659 & MAF & 0.317 & 0.267 & Allele & 0.583 & 1.277 & $0.533-3.058$ \\
\hline & $\mathrm{A}>\mathrm{G}$ & $\mathrm{A} / \mathrm{A}$ & $35(42.7)$ & $8(53.3)$ & & & & \\
\hline & & $\mathrm{A} / \mathrm{G}$ & $42(51.2)$ & $6(40.0)$ & Dominant & 0.445 & 1.535 & $0.508-4.632$ \\
\hline & & $\mathrm{G} / \mathrm{G}$ & $5(6.1)$ & $1(6.7)$ & Recessive & 1.000 & 0.909 & $0.099-8.382$ \\
\hline \multirow[t]{12}{*}{ ILI8 } & rs5744247 & MAF & 0.457 & 0.367 & Allele & 0.358 & 1.456 & $0.652-3.251$ \\
\hline & $\mathrm{C}>\mathrm{G}$ & $\mathrm{C} / \mathrm{C}$ & $24(29.3)$ & $5(33.3)$ & & & & \\
\hline & & $\mathrm{C} / \mathrm{G}$ & $41(50.0)$ & $9(60.0)$ & Dominant & 0.765 & 1.208 & $0.373-3.909$ \\
\hline & & $\mathrm{G} / \mathrm{G}$ & $17(20.7)$ & $1(6.7)$ & Recessive & 0.290 & 3.662 & $0.449-29.85$ \\
\hline & rs2043055 & MAF & 0.409 & 0.400 & Allele & 0.930 & 1.036 & $0.468-2.292$ \\
\hline & $A>G$ & $\mathrm{~A} / \mathrm{A}$ & $30(36.6)$ & $5(33.3)$ & & & & \\
\hline & & $\mathrm{A} / \mathrm{G}$ & $37(45.1)$ & $8(53.3)$ & Dominant & 1.000 & 0.867 & $0.271-2.775$ \\
\hline & & $\mathrm{G} / \mathrm{G}$ & $15(18.3)$ & $2(13.3)$ & Recessive & 1.000 & 1.455 & $0.297-7.138$ \\
\hline & rs7106524 & MAF & 0.396 & 0.433 & Allele & 0.704 & 0.859 & $0.391-1.886$ \\
\hline & $\mathrm{G}>\mathrm{A}$ & $\mathrm{G} / \mathrm{G}$ & $29(35.4)$ & $5(33.3)$ & & & & \\
\hline & & $\mathrm{G} / \mathrm{A}$ & $41(50.0)$ & $7(46.7)$ & Dominant & 1.000 & 0.914 & $0.285-2.930$ \\
\hline & & $\mathrm{A} / \mathrm{A}$ & $12(14.6)$ & $3(20.0)$ & Recessive & 0.697 & 0.686 & $0.168-2.796$ \\
\hline
\end{tabular}


Araki C (2016) The evidence of genetic polymorphisms of genes involved in the $P 2 R X 7$ signaling pathway as predictive biomarkers for response and loss of response to infliximab against Crohn's disease

\begin{tabular}{|c|c|c|c|c|c|c|c|c|}
\hline & rs 360717 & MAF & 0.104 & 0.200 & Allele & 0.133 & 0.463 & $0.166-1.290$ \\
\hline & $\mathrm{C}>\mathrm{G}$ & $\mathrm{C} / \mathrm{C}$ & $66(80.5)$ & $9(60.0)$ & & & & \\
\hline & & $\mathrm{C} / \mathrm{G}$ & $15(18.3)$ & $6(40.0)$ & Dominant & 0.082 & 0.364 & $0.113-1.170$ \\
\hline & & $\mathrm{G} / \mathrm{G}$ & $1(1.2)$ & $0(0)$ & Recessive & 1.000 & 0.571 & $0.022-14.67$ \\
\hline \multirow[t]{24}{*}{$I L 1 B$} & rs1143643 & MAF & 0.415 & 0.500 & Allele & 0.385 & 0.708 & $0.325-1.546$ \\
\hline & $\mathrm{G}>\mathrm{A}$ & $\mathrm{G} / \mathrm{G}$ & $27(32.9)$ & $4(26.7)$ & & & & \\
\hline & & $\mathrm{G} / \mathrm{A}$ & $42(51.2)$ & 7 (46.7) & Dominant & 0.768 & 0.741 & $0.216-2.543$ \\
\hline & & $\mathrm{A} / \mathrm{A}$ & $13(15.9)$ & $4(26.7)$ & Recessive & 0.293 & 0.518 & $0.143-1.880$ \\
\hline & rs1143633 & MAF & 0.396 & 0.500 & Allele & 0.289 & 0.657 & $0.301-1.434$ \\
\hline & $\mathrm{A}>\mathrm{G}$ & $\mathrm{A} / \mathrm{A}$ & $27(32.9)$ & $4(26.7)$ & & & & \\
\hline & & $\mathrm{A} / \mathrm{G}$ & $45(54.9)$ & $7(46.7)$ & Dominant & 0.768 & 0.741 & $0.216-2.543$ \\
\hline & & $\mathrm{G} / \mathrm{G}$ & $10(12.2)$ & $4(26.7)$ & Recessive & 0.222 & 0.382 & $0.102-1.432$ \\
\hline & rs3136558 & MAF & 0.470 & 0.467 & Allele & 0.977 & 1.012 & $0.464-2.207$ \\
\hline & $\mathrm{T}>\mathrm{C}$ & $\mathrm{T} / \mathrm{T}$ & $21(25.6)$ & $4(26.7)$ & & & & \\
\hline & & $\mathrm{T} / \mathrm{C}$ & $45(54.9)$ & $8(53.3)$ & Dominant & 1.000 & 1.056 & $0.303-3.676$ \\
\hline & & $\mathrm{C} / \mathrm{C}$ & $16(19.5)$ & $3(20.0)$ & Recessive & 1.000 & 0.970 & $0.244-3.846$ \\
\hline & rs1143630 & MAF & 0.152 & 0.100 & Allele & 0.580 & 1.619 & $0.456-5.744$ \\
\hline & $\mathrm{C}>\mathrm{A}$ & $\mathrm{C} / \mathrm{C}$ & $60(73.2)$ & $12(80.0)$ & & & & \\
\hline & & $\mathrm{C} / \mathrm{A}$ & $19(23.2)$ & $3(20.0)$ & Dominant & 0.753 & 1.467 & $0.378-5.692$ \\
\hline & & $\mathrm{A} / \mathrm{A}$ & $3(3.7)$ & $0(0)$ & Recessive & 1.000 & 1.365 & $0.067-27.780$ \\
\hline & rs16944 & MAF & 0.445 & 0.467 & Allele & 0.827 & 0.917 & $0.420-2.001$ \\
\hline & $\mathrm{G}>\mathrm{A}$ & $\mathrm{G} / \mathrm{G}$ & $26(31.7)$ & $4(26.7)$ & & & & \\
\hline & & $\mathrm{G} / \mathrm{A}$ & $29(47.6)$ & $8(53.3)$ & Dominant & 0.772 & 0.783 & $0.228-2.694$ \\
\hline & & $\mathrm{A} / \mathrm{A}$ & $17(20.7)$ & $3(20.0)$ & Recessive & 1.000 & 1.046 & $0.265-4.131$ \\
\hline & rs1143623 & MAF & 0.354 & 0.333 & Allele & 0.830 & 1.094 & $0.480-2.494$ \\
\hline & $\mathrm{G}>\mathrm{C}$ & $\mathrm{G} / \mathrm{G}$ & $35(42.7)$ & $7(46.7)$ & & & & \\
\hline & & $\mathrm{G} / \mathrm{C}$ & $36(43.9)$ & $6(40.0)$ & Dominant & 0.775 & 1.175 & $0.389-3.547$ \\
\hline & & $\mathrm{C} / \mathrm{C}$ & $11(13.4)$ & $2(13.3)$ & Recessive & 1.000 & 1.007 & $0.200-5.081$ \\
\hline
\end{tabular}

* Allele: allele model; Dominant: the minor allele dominant model; Recessive: the minor allele recessive model. (IFX, infliximab; CD, Crohn's disease; SNP, single nucleotide polymorphism; OR, odds ratio; CI, confidence interval; MAF, minor allele frequency).

of female gender showed response to IFX after 10 weeks of treatment. Subsequently, multivariate logistic regression analysis revealed that only the C/C genotype of rs11670259 in CARD8 independently contributed to response to IFX $(P=0.017, \mathrm{OR}=5.391$; Table 6$)$. Conversely, the $\mathrm{C} / \mathrm{T}$ or $\mathrm{T} / \mathrm{T}$ genotype of rs 11670259 in CARD8 contributed to primary non-response to IFX after 10 weeks of treatment.

\section{The gene-gene interaction in response to IFX after 1 year of treatment}

Likewise, in order to investigate the influence of the interaction of associated genetic factors on response to IFX after 1 year of treatment, multivariate logistic regression analysis indicated that three genetic factors, the $\mathrm{T} / \mathrm{T}$ or $\mathrm{T} / \mathrm{G}$ genotype of $\mathrm{rs} 3751143$ in $\mathrm{P} 2 \mathrm{RX}$, the $\mathrm{C} / \mathrm{C}$ genotype of rs4389238 in CARD8, and the $\mathrm{A} / \mathrm{G}$ or $\mathrm{G} / \mathrm{G}$ genotype of rs2282659 in CASP1, independently contributed to response to IFX ( $P$ $=0.012, \mathrm{OR}=6.379, P=0.013, \mathrm{OR}=5.114, P=0.004, \mathrm{OR}=7.803$, respectively; Table 7).

Conversely, the G/G genotype of rs3751143 in P2RX7, the C/T or T/T genotype of rs4389238 in CARD8, and the A/A genotype of rs2282659 in CASP1 independently contributed to loss of response to IFX after 1 year of treatment.

\section{Verification of genetic test to predict response to IFX after 10 weeks of treatment}

In order to predict response to IFX for CD patients after 10 weeks of treatment, genetic test was carried out using an independent genetic factor, the $\mathrm{C} / \mathrm{C}$ genotype of rs11670259 in CARD8, as a biomarker (Table 8). This test indicated that the sensitivity, specificity, positive predictive value, and negative predictive value were estimated to be at $68.1 \%, 63.6 \%, 95.2 \%$, and $15.9 \%$, respectively (Table 8 ).

\section{Verification of genetic test to predict response to IFX after 1 year of treatment}

Likewise, we performed genetic test with a combination of the three independent genetic factors as biomarkers to better predict response to IFX for CD patients after 1 year of treatment, indicating that the best combination of marker 6 (T/T or T/G genotype of rs3751143 in $P 2 R X 7$ and $\mathrm{A} / \mathrm{G}$ or $\mathrm{G} / \mathrm{G}$ genotype of rs2282659 in CASP1) was useful as a biomarker with the values of the highest scores of the $P$ value, OR, sensitivity, specificity, and positive predictive value (Table 9).

\section{Discussion}

This study is the first demonstration to report that the polymorphisms of CARD8, P2RX7, and CASP1 independently contribute to the therapeutic effect of IFX for CD patients.

From the pathophysiological perspective at 10 weeks after the start of IFX administration, the C/C genotype at rs11670259 in CARD8 may decrease the function of CARD8 in the genetic background, thereby leading to the diminution of the production of inflammatory cytokines as well as inflammatory mediators through the P2RX7 signaling pathway [14-19]. Therefore, not only the suppression of the TNFR signaling pathway due to IFX, but also the diminution of the P2RX7 signaling pathway due to this polymorphism may show good response to IFX after 10 weeks of treatment (Figure 2).

In contrast, the $\mathrm{C} / \mathrm{T}$ or $\mathrm{T} / \mathrm{T}$ genotype of rs11670259 in CARD8 may increase the function of CARD8 in the genetic background, 
Araki C (2016) The evidence of genetic polymorphisms of genes involved in the $P 2 R X 7$ signaling pathway as predictive biomarkers for response and loss of response to infliximab against Crohn's disease

Table 6. The interaction of genetic and environmental factors for response to IFX after 10 weeks of treatment for CD patients

\begin{tabular}{|c|c|c|}
\hline Factor & OR (95\% CI) & P value* \\
\hline C/C genotype of rs11670259 in CARD8 & 0.017 & $5.391(1.352-21.49)$ \\
\hline G/G genotype of rs1143623 in IL1B & $8.293(0.966-71.21)$ \\
\hline Female & 0.054 & $7.364(0.852-63.62)$ \\
\hline
\end{tabular}

* Allele: allele model; Dominant: the minor allele dominant model; Recessive: the minor allele recessive model. (IFX, infliximab; CD, Crohn's disease; SNP, single nucleotide polymorphism; OR, odds ratio; CI, confidence interval; MAF, minor allele frequency).

Table 7. Gene-gene interaction among P2RX7, CARD8, and CASP1 genotypes for response to IFX after 1 year of treatment for CD patients.

\begin{tabular}{|c|c|c|}
\hline Factor & OR (95\% CI) \\
\hline T/T or T/G genotype of rs3751143 in $P 2 R X 7$ & $6.379(1.498-27.17)$ \\
\hline C/C genotype of rs4389238 in CARD8 & $5.114(1.416-18.48)$ \\
\hline A/G or G/G genotype of rs2282659 in CASPI & 0.012 \\
\hline
\end{tabular}

*Factors were statistically analyzed by multivariate logistic regression analysis.( IFX, infliximab; CD, Crohn's disease; OR, odds ratio; CI, confidence interval).

Table 8. Genetic factor determined by genetic test for response to IFX after 10 weeks of treatment for CD patients.

\begin{tabular}{|c|c|c|c|c|}
\hline \multirow{2}{*}{ Biomarker } & Statistical results & \multicolumn{2}{|c|}{ Genetic diagnosis } \\
\cline { 2 - 5 } & OR (95\% CI) & P value* & sensitivity & specificity \\
\hline C/C genotype of rs11670259 in CARD8 & $3.736(1.029-13.57)$ & 0.047 & 68.1 & 63.6 \\
\hline
\end{tabular}

*Factors were statistically analyzed by multivariate logistic regression analysis. (IFX, infliximab; CD, Crohn's disease; OR, odds ratio; CI, confidence interval).

Table 9. Combination of genetic factors determined by genetic test for response to IFX after 1 year of treatment for CD patients. *Factors were statistically analyzed by Fisher's exact test. (IFX, infliximab; CD, crohn's disease; OR, odds ratio; CI, confidence interval; PPV, positive predictive value; NPV, negative predictive value)

\begin{tabular}{|c|c|c|c|c|c|c|c|c|c|}
\hline \multirow[t]{2}{*}{ Biomarker } & \multirow{2}{*}{$\begin{array}{c}P 2 R X 7 \\
\text { rs3751143 }\end{array}$} & \multirow{2}{*}{$\begin{array}{c}C A R D 8 \\
\text { rs4389238 }\end{array}$} & \multirow{2}{*}{$\begin{array}{c}C A S P 1 \\
\text { rs2282659 }\end{array}$} & \multicolumn{2}{|c|}{ Statistical results } & \multicolumn{4}{|c|}{ Genetic diagnosis } \\
\hline & & & & OR (95\% CI) & $P$ value* & sensitivity & specificity & PPV & NPV \\
\hline marker 1 & $\mathrm{~T} / \mathrm{T}$ or $\mathrm{T} / \mathrm{G}$ & - & - & $7.000(1.961-24.99)$ & 0.004 & 93.8 & 31.6 & 87.5 & 50.0 \\
\hline marker 2 & - & $\mathrm{C} / \mathrm{C}$ & - & $4.333(1.341-14.01)$ & 0.012 & 53.6 & 78.9 & 92.9 & 25.0 \\
\hline marker 3 & - & - & $\mathrm{A} / \mathrm{G}$ or $\mathrm{G} / \mathrm{G}$ & $6.698(1.831-24.50)$ & 0.002 & 55.7 & 84.2 & 94.7 & 27.1 \\
\hline marker 4 & $\mathrm{~T} / \mathrm{T}$ or $\mathrm{T} / \mathrm{G}$ & $\mathrm{C} / \mathrm{C}$ & - & $5.444(1.490-19.90)$ & 0.006 & 50.5 & 84.2 & 94.2 & 25.0 \\
\hline marker 5 & - & $\mathrm{C} / \mathrm{C}$ & $\mathrm{A} / \mathrm{G}$ or $\mathrm{G} / \mathrm{G}$ & $6.592(0.837-51.91)$ & 0.071 & 26.8 & 94.7 & 96.3 & 20.2 \\
\hline marker 6 & $\mathrm{~T} / \mathrm{T}$ or $\mathrm{T} / \mathrm{G}$ & - & $\mathrm{A} / \mathrm{G}$ or $\mathrm{G} / \mathrm{G}$ & $9.424(2.064-43.04)$ & 0.001 & 52.6 & 89.5 & 96.2 & 27.0 \\
\hline marker 7 & $\mathrm{~T} / \mathrm{T}$ or $\mathrm{T} / \mathrm{G}$ & $\mathrm{C} / \mathrm{C}$ & $\mathrm{A} / \mathrm{G}$ or $\mathrm{G} / \mathrm{G}$ & $6.250(0.792-49.28)$ & 0.069 & 25.8 & 94.7 & 96.2 & 20.0 \\
\hline
\end{tabular}

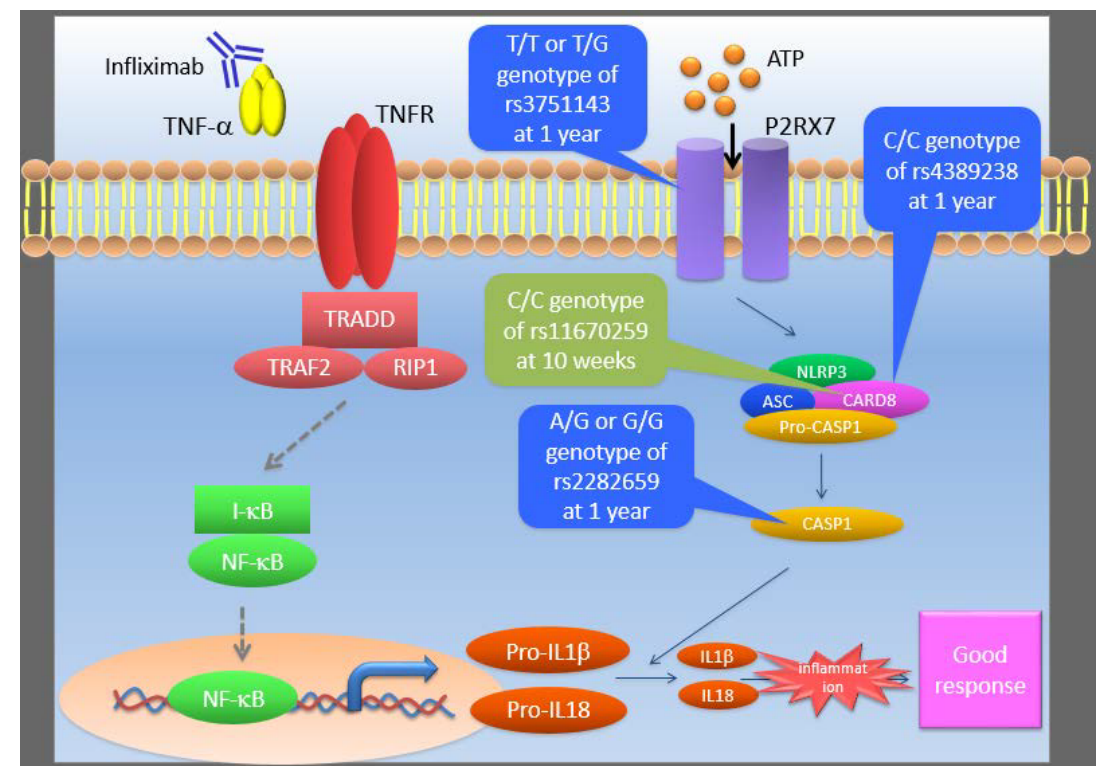

Figure 2. The putative mechanism of good response to infliximab for CD patients.

A schematic representation indicates the putative mechanism of good response to infliximab after 10 weeks and 1 year of treatment. Ligands, TNF- $\alpha$ and ATP, activate its receptors, TNFR and P2RX7, respectively. Subsequently the downstream signals in each signaling pathway induce the production of inflammatory cytokines including IL-1 $\beta$ and IL-18. In the CD patients with the present polymorphisms of $P 2 R X 7, C A R D 8$, and $C A S P 1$, the signals in the P2RX7 pathway may be diminished in the genetic background. Whereas, the signals in the TNFR pathway can be suppressed by infliximab.

Abbreviations: TNF, tumor necrosis factor; TNFR, TNF receptor; TRADD, tumor necrosis factor receptor type 1-associated DEATH domain protein; TRAF2, TNF receptor-associated

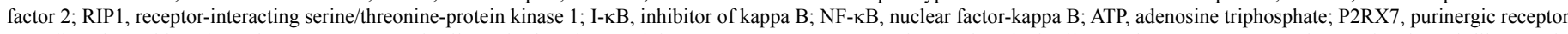
$\mathrm{P} 2 \mathrm{X}$, ligand gated ion channel, 7; NLRP3, NLR family pyrin domain containing 3; CARD8, caspase recruitment domain family member 8; ASC, apoptosis-associated speck-like protein containing a carboxy-terminal CARD; CASP1, caspase 1; IL-1 $\beta$, interleukin 1 beta; IL-18, interleukin 18. 
thereby leading to the acceleration of the production of inflammatory cytokines and mediators through the P2RX7 signaling pathway. The condition with the elevated production of inflammatory cytokines and mediators through the activation of the P2RX7 signaling pathway due to this polymorphism may predominate over that with the suppressed production of inflammatory cytokines and mediators through the TNFR signaling pathway due to IFX. Therefore, the CD patients under such conditions may eventually result in primary non-response after 10 weeks of treatment (Figure 3).

At 1 year after the start of IFX administration, the T/T or T/G genotype of rs3751143 in $P 2 R X 7$, the C/C genotype of rs4389238 in CARD8, or A/G or G/G genotype at rs2282659 in CASP1 may slightly reduce the function of P2RX7, CARD8, and CASP1 in the genetic background, thereby leading to a decrease in the production of inflammatory cytokines and mediators through the P2RX7 signaling pathway. As similar to the mechanism observed after 10 weeks of treatment, in these CD patients, both the diminution of the P2RX7 signaling pathway due to these polymorphisms and the suppression of the TNFR signaling pathway due to IFX may show good response to IFX after 1 year of treatment (Figure 2).

Conversely, the G/G genotype of rs3751143 in P2RX7, the C/T or $\mathrm{T} / \mathrm{T}$ genotype of rs4389238 in CARD8, and A/A genotype at rs2282659 in CASP1 may slightly accelerate the function of P2RX7, CARD8, and CASP1 in the genetic background, thereby leading to an increase in the production of inflammatory cytokines and mediators through the P2RX7 signaling pathway. Therefore, not only the elevated production of inflammatory cytokines and mediators through the accelerated P2RX7 signaling pathway due to these polymorphisms, but also a decrease in the suppressed production of inflammatory cytokines and mediators through the TNFR signaling pathway due to the reduction of the IFX actions, may exacerbate inflammation of the intestines in the patients and eventually lead to secondary loss of response to IFX after 1 year of treatment (Figure 3). Although the decisive factors have not yet been identified, the reduction of the IFX actions after 1 year of treatment may be caused by various clinical risk factors, including a shortened half-life of IFX due to the increased clearance of IFX, the dominant mechanism of inflammation, the production of antibodies to IFX (ATI), and the low serum concentrations of the IFX levels (up to $60 \%)[29,30]$. Indeed, approximately $15-61 \%$ of CD patients developed ATI [31-33], although ATI was not examined in this study. Of course, since these CD patients, who showed secondary loss of response after 1 year of treatment, showed response to IFX at the 10 -week treatment, at 10 weeks after the start of IFX administration, the condition with the suppressed production of inflammatory cytokines and mediators through the TNFR signaling pathway due to IFX may predominate over that with the slightly elevated production of inflammatory cytokines and mediators through the activation of the P2RX7 signaling pathway due to the polymorphisms in the genetic background.

With regard to genetic test with the IFX-related polymorphisms, the $\mathrm{C} / \mathrm{C}$ genotype at rs11670259 in CARD8 is useful as a biomarker to predict response to IFX for CD patients after 10 weeks of treatment with significant differences (Table 8). As this test showed the sensitivity of $68.1 \%$, we hypothesize that the activation of the P2RX7 signaling pathway may contribute to inflammation of the intestines in about two-thirds of the CD patients who showed good response to IFX after 10 weeks of treatment. Moreover, the positive predictive value of this test was very higher at $95.2 \%$, thereby indicating the very higher probability that almost all of the $\mathrm{CD}$ patients with the $\mathrm{C} / \mathrm{C}$ genotype of rs11670259 in CARD8 could show good response to IFX after 10 weeks of treatment.

On the other hand, after 1 year of treatment, genetic test showed the combination marker 6 (T/T or T/G genotype of rs3751143 in P2RX7

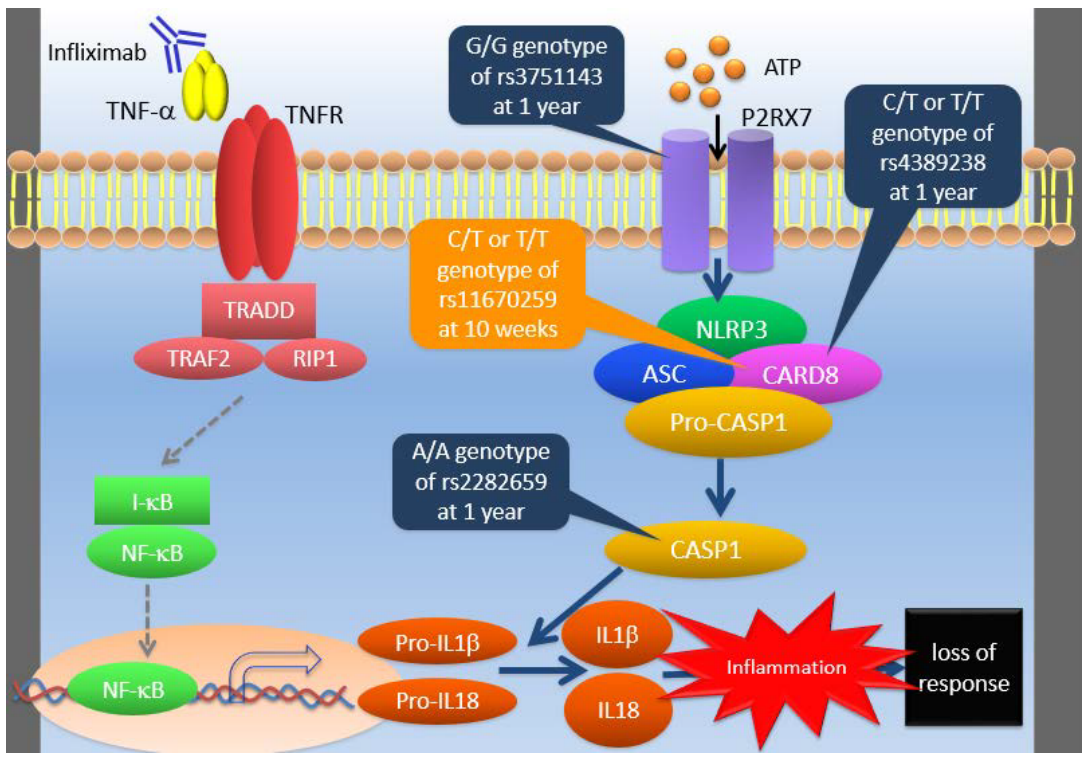

Figure 3. The putative mechanism of loss of response to infliximab for $\mathrm{CD}$ patients

A schematic representation indicates the putative mechanism of primary non-response and secondary loss of response to infliximab after 10 weeks and 1 year of treatment, respectively. In the CD patients with the present polymorphisms of $P 2 R X 7, C A R D 8$, and $C A S P 1$, the signals in the P2RX7 pathway may be slightly activated in the genetic background. Whereas, the signals in the TNFR pathway can be suppressed by infliximab.

Abbreviations: TNF, tumor necrosis factor; TNFR, TNF receptor; TRADD, tumor necrosis factor receptor type 1-associated DEATH domain protein; TRAF2, TNF receptor-associated factor 2; RIP1, receptor-interacting serine/threonine-protein kinase 1; I- $\kappa \mathrm{B}$, inhibitor of kappa B; NF- $\kappa \mathrm{B}$, nuclear factor-kappa B; ATP, adenosine triphosphate; P2RX7, purinergic receptor P2X, ligand gated ion channel, 7; NLRP3, NLR family pyrin domain containing 3; CARD8, caspase recruitment domain family member 8; ASC, apoptosis-associated speck-like protein containing a carboxy-terminal CARD; CASP1, caspase 1; IL-1 $\beta$, interleukin 1 beta; IL-18, interleukin 18. 
Araki C (2016) The evidence of genetic polymorphisms of genes involved in the $P 2 R X 7$ signaling pathway as predictive biomarkers for response and loss of response to infliximab against Crohn's disease

and $\mathrm{A} / \mathrm{G}$ or G/G genotype of rs2282659 in CASP1) could be useful as a biomarker to predict response to IFX for CD patients with significant differences (Table 9). As similar to the mechanism observed after 10 weeks of treatment, the sensitivity of $52.6 \%$ was seen in the genetic test after 1 year of treatment, indicating that the activation of the P2RX7 signaling pathway may contribute to inflammation of the intestines in about half of all CD patients who showed good response to IFX after 1 year of treatment. Likewise, the positive predictive value of $96.2 \%$ shown in the test indicates the very higher probability that almost all of the CD patients with both the T/T or T/G genotype of rs 3751143 in $P 2 R X 7$ and $A / G$ or $G / G$ genotype of rs2282659 in CASP1 could show good response to IFX after 1 year of treatment.

In the patients who show secondary loss of response to IFX, remission can be managed by shortening interval between dosing [34], dose intensification [35], and/or switching to other biological agents [36,37]. For example, adalimumab is a fully humanized monoclonal antibody approved for the treatment of CD patients who have failed to respond to conventional agents and anti-TNF- $\alpha$ therapies $[30,36]$. Certolizumab pegol is a pegylated humanized monoclonal Fab' fragment that binds to TNF- $\alpha[30,37,38]$. Natalizumab is a humanized monoclonal antibody against $\alpha 4$ integrin $[39,40]$. When these agents are chosen for the treatment of $\mathrm{CD}$, then useful biomarkers to predict response or loss of response to IFX would be required.

Finally, not only the antagonists of P2RX7 [14,20], but also other molecules involved in the P2RX7 signaling pathway could be targets for newly developed therapeutic agents to combat primary non-response and secondary loss of response to IFX for CD patients.

\section{Conclusion}

This is the first report to show that CARD8 is related to response and primary non-response to IFX after 10 weeks of treatment, and $P 2 R X 7, C A R D 8$, and CASP1 are related to response and secondary loss of response to IFX after 1 year of treatment for Japanese CD patients. The polymorphism, the $\mathrm{C} / \mathrm{C}$ genotype at rs11670259 in CARD8, and the combination polymorphisms, T/T or T/G genotype of rs 3751143 in $P 2 R X 7$ and $\mathrm{A} / \mathrm{G}$ or $\mathrm{G} / \mathrm{G}$ genotype of rs2282659 in CASP1, were found to useful as biomarkers to predict response to IFX after 10 weeks and 1 year of treatment, respectively. These molecules including P2RX7, CARD8, and CASP1 in the P2RX7 signaling pathway could therefore become targets for new therapeutic drugs and thereby help patients to overcome primary non-response and secondary loss of response to IFX in $\mathrm{CD}$ patients.

\section{Acknowledgement}

We are grateful to the physicians and CD patients for participating in this study. This work was supported by a Grant-in-Aid for Scientific Research (C) (KAKENHI No. 16K08912) from the Ministry of Education, Culture, Sports, Science and Technology of Japan (K. Tsukamoto) and a research grant from the Non-Profit Organization Aimed to Support Community Medicine Research in Nagasaki, Japan (K. Tsukamoto).

\section{Conflicts of interest}

The authors declare that they have no competing interests in association with this study.

\section{References}

1. Xavier RJ, Podolsky DK (2007) Unravelling the pathogenesis of inflammatory bowel disease. Nature 448: 427-434. [Crossref]
2. Cho JH (2008) The genetics and immunopathogenesis of inflammatory bowel disease. Nat Rev Immunol 8: 458-466. [Crossref]

3. Abraham C, Cho JH (2009) Inflammatory bowel disease. $N$ Engl J Med 361: 20662078. [Crossref]

4. Lichtenstein GR, Hanauer SB, Sandborn WJ; Practice Parameters Committee of American College of Gastroenterology (2009) Management of Crohn's disease in adults. Am J Gastroenterol 104: 465-483. [Crossref]

5. Ueno F, Matsui T, Matsumoto T, Matsuoka K, Watanabe M, et al. (2013) Evidencebased clinical practice guidelines for Crohn's disease, integrated with formal consensus of experts in Japan. J Gastroenterol 48: 31-72. [Crossref]

6. van Deventer SJ (1999) Review article: targeting TNF alpha as a key cytokine in the inflammatory processes of Crohn's disease--the mechanisms of action of infliximab. Aliment Pharmacol Ther 13 Suppl 4: 3-8. [Crossref]

7. Hanauer SB, Feagan BG, Lichtenstein GR, Mayer LF, Schreiber S, et al. (2002) Maintenance infliximab for Crohn's disease: the ACCENT I randomised trial. Lancet 359: 1541-1549. [Crossref]

8. Rooryck C, Barnetche T, Richez C, Laleye A, Arveiler B, et al. (2008) Influence of FCGR3A-V212F and TNFRSF1B-M196R genotypes in patients with rheumatoid arthritis treated with infliximab therapy. Clin Exp Rheumatol 26: 340-342. [Crossref]

9. Cañete JD, Suárez B, Hernández MV, Sanmartí R, Rego I, et al. (2009) Influence of variants of Fc gamma receptors IIA and IIIA on the American College of Rheumatology and European League Against Rheumatism responses to anti-tumour necrosis factor alpha therapy in rheumatoid arthritis. Ann Rheum Dis 68: 1547-1552.

10. Tan RJL, Gibbons LJ, Potter C, Hyrich KL, Morgan AW, et al. (2010) Investigation of rheumatoid arthritis susceptibility genes identifies association of AFF3 and CD226 variants with response to anti-tumour necrosis factor treatment. Ann Rheum Dis 69: 1029-1035.

11. Cui J, Saevarsdottir S, Thomson B, Padyukov L, van der Helm-van Mil AHM, et al. (2010) Rheumatoid arthritis risk allele PTPRC is also associated with response to antitumor necrosis factor? therapy. Arthritis Rheum 62: 1849-1861.

12. Coulthard LR, Taylor JC, Eyre S; Biologics in Rheumatoid Arthritis Genetics and Genomics, Robinson JI, Wilson AG, et al. (2011) Genetic variants within the MAP kinase signalling network and anti-TNF treatment response in rheumatoid arthritis patients. Ann Rheum Dis 70: 98-103. [Crossref]

13. Urabe S, Isomoto $H$, Ishida $T$, Maeda $K$, Inamine $T$, et al. (2015) Genetic polymorphisms of IL-17F and TRAF3IP2 could be predictive factors of the long-term effect of Infliximab against Crohn's disease. Biomed Res Int 4: 1-12

14. Mehta N, Kaur M, Singh M, Chand S, Vyas B, et al. (2014) Purinergic receptor P2Xa: a novel target for anti-inflammatory therapy. Bioorg Med Chem 22: 54-88. [Crossref]

15. Sim JA, Young MT, Sung HY, North RA, Surprenant A (2004) Reanalysis of P2X7 receptor expression in rodent brain. $J$ Neurosci 24: 6307-6314. [Crossref]

16. Kahlenberg JM, Dubyak GR (2004) Mechanisms of caspase-1 activation by P2X7 receptor-mediated K+ release. Am J Physiol Cell Physiol 286: C1100-1108. [Crossref]

17. Mehta VB, Hart J, Wewers MD (2001) ATP-stimulated release of interleukin (IL)1 beta and IL-18 requires priming by lipopolysaccharide and is independent of caspase-1 cleavage. J Biol Chem 276: 3820-3826. [Crossref]

18. Bulanova E, Budagian V, Orinska Z, Hein M, Petersen F, et al. (2005) Extracellular ATP induces cytokine expression and apoptosis through $\mathrm{P} 2 \mathrm{X} 7$ receptor in murine mast cells. J Immunol 174: 3880-3890. [Crossref]

19. Ferrari D, Wesselborg S, Bauer MKA, Schulze-Osthoff K (1997) Extracellular ATP activates transcription factor NF-?B through the P2Z purinoreceptor by selectively targeting NF-?B p65 (RelA). J Cell Biol 139: 1635-1643.

20. McCulloch CA, Downey GP, El-Gabalawy H (2006) Signalling platforms that modulate the inflammatory response: new targets for drug development. Nat Rev Drug Discov 5: 864-876. [Crossref]

21. Fantuzzi G, Ku G, Harding MW, Livingston DJ, Sipe JD, et al. (1997) Response to local inflammation of IL-1 beta-converting enzyme- deficient mice. J Immunol 158 1818-1824. [Crossref]

22. Solle M, Labasi J, Perregaux DG, Stam E, Petrushova N, et al. (2001) Altered cytokine production in mice lacking P2X(7) receptors. J Biol Chem 276: 125-132. [Crossref]

23. Labasi JM, Petrushova N, Donovan C, McCurdy S, Lira P, et al. (2002) Absence of the $\mathrm{P} 2 \mathrm{X} 7$ receptor alters leukocyte function and attenuates an inflammatory response. J Immunol 168: 6436-6445. 
Araki C (2016) The evidence of genetic polymorphisms of genes involved in the $P 2 R X 7$ signaling pathway as predictive biomarkers for response and loss of response to infliximab against Crohn's disease

24. Schoultz I, Verma D, Halfvarsson J, Törkvist L, Fredrikson M, et al. (2009) Combined polymorphisms in genes encoding the inflammasome components NALP3 and CARD8 confer susceptibility to Crohn's disease in Swedish men. Am J Gastroenterol 104: 1180-1188. [Crossref]

25. Kurashima Y, Amiya T, Nochi T, Fujisawa K, Haraguchi T, et al. (2012) Extracellular ATP mediates mast cell-dependent intestinal inflammation through $\mathrm{P} 2 \mathrm{X} 7$ purinoceptors. Nat Commun 3: 1034. [Crossref]

26. Best WR, Becket JM, Singleton JW, Kern F Jr (1976) Development of a Crohn's disease activity index: National Cooperative Crohn's Disease Study. Gastroenterology 70: 439-444.

27. Nanashima K, Mawatari T, Tahara N, Higuchi N, Nakaura A, et al. (2012) Genetic variants in antioxidant pathway: risk factors for hepatotoxicity in tuberculosis patients. Tuberculosis (Edinb) 92: 253-259. [Crossref]

28. Mawatari T, Yoshida E, Higuchi N, Sato K, Inamine T, et al. (2016) A combination polymorphism of the glutathione synthesis genes can be a predictive biomarker for anti-tuberculosis drug-induced hepatotoxicity in Japanese patients with pulmonary tuberculosis. $J$ Lung Dis and Treat 2: 105.

29. Maharshak N, Plevy SE (2011) A Crohn's disease patient who does not respond to infliximab: what is next? Clin Gastroenterol Hepatol 9: 1033-1038. [Crossref]

30. Yanai H, Hanauer SB (2011) Assessing response and loss of response to biological therapies in IBD. Am J Gastroenterol 106: 685-698. [Crossref]

31. Baert F, Noman M, Vermeire S, Van Assche G, D' Haens G, et al. (2003) Influence of immunogenicity on the long-term efficacy of infliximab in Crohn's disease. $N$ Engl J Med 348: 601-608. [Crossref]

32. Hanauer SB, Wagner CL, Bala M, Mayer L, Travers S, et al. (2004) Incidence and importance of antibody responses to infliximab after maintenance or episodic treatment in Crohn's disease. Clin Gastroenterol Hepatol 2: 542-553. [Crossref]

33. Ungar B, Chowers Y, Yavzori M, Picard O, Fudim E, et al. (2014) The tempora evolution of antidrug antibodies in patients with inflammatory bowel disease treated with infliximab. Gut 63: 1258-1264. [Crossref]

34. Kopylov U, Mantzaris GJ, Katsanos KH, Reenaers C, Ellul P, et al. (2011) The efficacy of shortening the dosing interval to once every six weeks in Crohn's patients losing response to maintenance dose of infliximab. Aliment Pharmacol Ther 33: 349-357.

35. Gisbert JP, Panés J (2009) Loss of response and requirement of infliximab dose intensification in Crohn's disease: a review. Am J Gastroenterol 104: 760-767.

36. Sandborn WJ, Rutgeerts P, Enns R, Hanauer SB, Colombel JF, et al. (2007) Adalimumab induction therapy for Crohn disease previously treated with infliximab: a randomized trial. Ann Intern Med 146: 829-838. [Crossref]

37. Sandborn WJ, Abreu MT, D'Haens G, Colombel JF, Vermeire S, et al. (2010) Certolizumab pegol in patients with moderate to severe Crohn's disease and secondary failure to infliximab. Clin Gastroenterol Hepatol 8: 688-695. [Crossref]

38. Sandborn WJ, Feagan BG, Stoinov S, Honiball PJ, Rutgeerts P, et al. (2007) Certolizumab pegol for the treatment of Crohn's disease. N Engl J Med 357: 228-238. [Crossref]

39. Sandborn WJ, Colombel JF, Enns R, Feagan BG, Hanauer SB, et al. (2005) Natalizumab induction and maintenance therapy for Crohn's disease. N Engl J Med 353: 1912-1925. [Crossref]

40. Targan SR, Feagan BG, Fedorak RN, Lashner BA, Panaccione R, et al. (2007) Natalizumab for the treatment of active Crohn's disease: results of the ENCORE Trial. Gastroenterology 132: 1672-1683. [Crossref].

Copyright: (C2016 Araki C. This is an open-access article distributed under the terms of the Creative Commons Attribution License, which permits unrestricted use, distribution, and reproduction in any medium, provided the original author and source are credited. 